\title{
SUR LES ORBITES D'UN SOUS-GROUPE SPHÉRIQUE DANS LA VARIÉTÉ DES DRAPEAUX
}

\author{
PAR NicOlas RESSAYRE
}

\begin{abstract}
RÉsumÉ. - Soient $G$ un groupe algébrique complexe réductif et connexe, $B$ un sous-groupe de Borel de $G$ et $H$ un sous-groupe sphérique de $G$.

Soit $X$ un plongement $G \times G$-équivariant de $G$. Nous savons que $B \times H$ n'a qu'un nombre fini d'orbites dans $G$; nous montrons qu'il n'en a qu'un nombre fini dans $X$. Soit $\bar{V}$ l'adhérence dans $X$ d'une orbite de $B \times H$ dans $G$ et $\overline{\mathcal{O}}$ l'adhérence d'une orbite de $G \times G$ dans $X$. Si $X$ est torö̈dal, nous montrons que l'intersection $\bar{V} \cap \overline{\mathcal{O}}$ est propre dans $X$ et la décrivons ensemblistement. Si de plus $X$ est lisse, nous calculons les multiplicités d'intersections qui sont des puissances de 2 . Enfin, si $X$ est toroïdal, lisse et complet, nous exprimons la classe de cohomologie de $\bar{V}$ comme une combination linéaire des classes d'adhérence dans $X$ d'orbites de $B \times B$ dans $G$. Nous utilisons la cohomologie $B$-équivariante pour obtenir ce dernier résultat.

Soit $Y$ un plongement lisse $G$-équivariant et toroïdal de $G / H$ et $\overline{\mathcal{O}}$ l'adhérence d'une orbite de $G$ dans $Y$. Soit $\bar{V}$ l'adhérence dans $Y$ d'une orbite de $B$ dans $G / H$. Dans [4], après la proposition $6, \mathrm{M}$. Brion demande si chaque composante irréductible de $\bar{V} \cap \overline{\mathcal{O}}$ contient des points lisses de $\bar{V}$ : nous répondons négativement à cette question dans la dernière partie.
\end{abstract}

Texte reçu le 2 décembre 2002, révisé le 3 novembre 2003, accepté le 16 juin 2003

Nicolas Ressayre, Université Montpellier II, Département de Mathématiques, Case courrier 051, Place Eugène Bataillon, 34095 Montpellier Cedex 5 (France)

E-mail : ressayre@math.univ-montp2.fr

Classification mathématique par sujets (2000). — 14M15, 14M17, 14C17, 55N91.

Mots clefs. - Plongement de groupes, variétés sphériques, adhérences d'orbites, variété des drapeaux, cohomologie équivariante . 


\begin{abstract}
On the orbits of a spherical subgroup in the flag manifold)
Let $G$ be a complex reductive algebraic group, $B$ be a Borel subgroup of $G$ and $H$ be a spherical subgroup of $G$.

Let $X$ be a $G \times G$-equivariant embedding of $G$. We know that $B \times H$ have finitely many orbits in $G$; we show that it has finitely many ones in $X$. Let $\bar{V}$ be the closure in $X$ of a $(B \times H)$-orbit in $G$, and $\overline{\mathcal{O}}$ be the closure of a $(G \times G)$-orbit in $X$. If $X$ is toroïdal, we show that the intersection $\bar{V} \cap \overline{\mathcal{O}}$ is proper in $X$ and we describe this intersection. If in addition $X$ is smooth, we determine the intersection multiplicities of $\bar{V} \cap \overline{\mathcal{O}}$, which are powers of 2 . If $X$ is toroïdal, smooth and complete, we write the class of cohomology of $\bar{V}$ as a linear combinaison of the classes of the closures in $X$ of the $(B \times B)$-orbits in $G$. The proof of this last statement uses $B$-equivariant cohomology. Let $Y$ be a smooth $G$-equivariant embedding of $G / H$ and $\overline{\mathcal{O}}$ be the closure of a $G$-orbit in $Y$. Let $\bar{V}$ be the closure in $Y$ of a $B$-orbit in $G / H$. In [4], just after Proposition 6 , M. Brion asks if each irreducible component of $\bar{V} \cap \overline{\mathcal{O}}$ intersects the set of the smooth points in $\bar{V}$ : we give an example which answers 'no' to this question.
\end{abstract}

\title{
1. Introduction
}

Soient $G$ un groupe algébrique complexe réductif et connexe, et $B$ un sousgroupe de Borel de $G$. Soit $H$ un sous-groupe fermé de $G$. On suppose que $H$ est sphérique c'est-à-dire (voir [1]) que l'ensemble $\mathbf{H}(G / B)$ des orbites de $H$ dans $G / B$ est fini. Ces orbites et leurs adhérences jouent un rôle important en théorie des représentations (voir par exemple [20]). Par ailleurs, les classes des adhérences d'orbites de $B$ dans les $G$-variétés sphériques (c'est-à-dire les variétés normales, munies d'une action de $G$ et ne contenant qu'un nombre fini d'orbites de $B$ ) sont des générateurs naturels de la cohomologie de ces variétés, supposées lisses et complètes. Il est donc intéressant de comprendre l'ensemble $\mathbf{B}(G / H)$ des orbites de $B$ dans $G / H$. Ce dernier est en bijection naturelle avec $\mathbf{H}(G / B)$.

Fixons un tore maximal $T$ de $G$ inclus dans $B$. Si $V$ est une orbite de $B$ dans $G / H$, on note $\bar{V}$ son adhérence dans $G / H$. On construit dans [14], [9] et [4] un graphe orienté $\Gamma(G / H)$ dont les sommets sont les éléments de $\mathbf{B}(G / H)$ et dont les arêtes, qui peuvent être simples ou doubles, sont étiquetées par les racines simples de $G$. Soit $\alpha$ une racine simple et $P_{\alpha}$ le sous-groupe parabolique minimal de $G$ et contenant $B$ associé à $\alpha$. Soit $V$ et $V^{\prime}$ deux orbites de $B$ dans $G / H$. Une arête étiquetée par $\alpha$ monte de $V$ à $V^{\prime}$ si et seulement si $\bar{V} \neq P_{\alpha} \cdot \bar{V}=\bar{V}^{\prime}$. Alors, l'application naturelle $P_{\alpha} \times{ }_{B} \bar{V} \rightarrow \bar{V}^{\prime}$ est génériquement finie de degré 1 ou 2 ; ceci détermine si l'arête est simple ou double.

Des travaux de M. Brion mais aussi de S. Pin ont montré que ce graphe $\Gamma(G / H)$ apporte des informations sur la lissité, la normalité, la classe de cohomologie des adhérences d'orbites de $H$ dans $G / B$ (voir [3], [4] et [11]). Comprendre les interactions entre les propriétés combinatoires, géométriques et topologiques de l'ensemble $\mathbf{H}(G / B)$ est un sujet vaste auquel cet article apporte une modeste contribution.

TOME $132-2004-\mathrm{N}^{\mathrm{O}} 4$ 
On considère l'action de $G \times G$ sur $G$ par multiplication à gauche et à droite. L'ensemble $\mathbf{B}(G / H)$ est en bijection naturelle avec l'ensemble $\mathbf{B H}(G)$ des orbites de $B \times H$ dans $G$. On utilisera implicitement dans cette introduction les bijections naturelles entre les trois ensembles $\mathbf{H}(G / B), \mathbf{B}(G / H)$ et $\mathbf{B H}(G)$. On s'intéresse aux éléments de $\mathbf{B H}(G)$ et surtout à leurs adhérences dans les plongements $G \times G$-équivariant de $G$. Soit $X$ un tel plongement. Notre premier résultat (voir le corollaire 4.7) est le

ThÉORÈme A. - L'ensemble des orbites de $B \times H$ dans $X$ est fini.

Lorsque $H=B$, ce résultat était déjà connu puisque plus généralement, toute variété sphérique ne contient qu'un nombre fini d'orbite d'un sous-groupe de Borel. Simultanément à la rédaction du présent article, T.A. Springer [18] a paramétré les orbites de $B \times B$ dans le plongement canonique de $G$ (supposé semi-simple adjoint). Ici, dans le cas où $X$ est toroïdal (voir le paragraphe 4.1.1 pour une définition précise), nous paramétrons (voir la proposition 4.6) les orbites de $B \times H$ dans $X$.

Soit $V_{G}$ dans $\mathbf{B H}(G)$. Notons $\bar{V}_{G}^{X}$ l'adhérence de $V_{G}$ dans $X$. Nous obtenons des résultats sur les sous-variétés $\bar{V}_{G}^{X}$ dès que $X$ est torö̈dal. Cependant, par soucis de simplicité, nous supposons dans l'introduction, que $G$ est semi-simple adjoint et que $X$ est le plongement canonique de $G$ (voir [5]). Notons $Z$ l'unique orbite fermée de $G \times G$ dans $X$. Celle-ci est isomorphe à $G / B \times G / B$, ou encore à $G / B^{-} \times G / B$ où $B^{-}$est le sous-groupe de Borel de $G$ contenant $T$ et opposé à $B$.

Nous avons encore besoin de notations pour énoncer nos résultats. Soit $W$ le groupe de Weyl de $T$ et $U$ le radical unipotent de $B$. Pour tout $w$ dans $W$, on pose $U_{w}=U \cap w U w^{-1}$ et $B_{w}=B \cap w B w^{-1}$.

Considérons un chemin $\gamma$ de $\Gamma(G / H)$ qui monte à partir du sommet correspondant à $V_{G}$. Soit $V_{G / B}^{\prime}$ l'orbite de $H$ dans $G / B$ associée au sommet le plus haut de $\gamma$. On note $D(\gamma)$ le nombre d'arêtes doubles le long de $\gamma$ et $w(\gamma)$ le produit dans $W$ des réflexions simples associées aux étiquettes de $\gamma$. Le théorème 4.9 décrit un ouvert de $\bar{V}_{G}^{X}$ associé à $w(\gamma)$. Dans le contexte de l'introduction, on obtient le

ThÉORÈme B. - On reprend les notations ci-dessus. Alors, il existe un ouvert $\Omega_{V, w(\gamma)}$ de $\bar{V}_{G}^{X}$ stable par $B_{w(\gamma)} \times H$ et une fibration localement triviale $B_{w(\gamma)^{-}}$ invariante et $H$-équivariante $\psi: \Omega_{V, w(\gamma)} \rightarrow V_{G / B}^{\prime}$.

De plus, il existe une sous-variété $S_{V, w(\gamma)}$ de $\Omega_{V, w(\gamma)}$ stable par $T$, telle que l'action de $U_{w(\gamma)}$ induise un isomorphisme de $U_{w(\gamma)} \times S_{V, w(\gamma)}$ sur la fibre de $\psi$.

Enfin, la variété $S_{V, w(\gamma)}$ a $2^{D(\gamma)}$ composantes irréductibles; chacune est isomorphe à l'espace affine de dimension rang de $G$ et intersecte $Z$ suivant l'unique point fixe de $B^{-} \times B$ dans $Z$.

BULletin DE LA SOCiÉtÉ MATHÉMATIQUE DE FRANCE 
Le théorème 4.10 décrit l'intersection de $\bar{V}_{G}^{X}$ et de l'adhérence d'une orbite de $G \times G$ dans $X$. Dans le cadre de l'introduction, ce dernier implique le

ThÉORÈme C. - Rappelons que $Z$ est canoniquement isomorphe à $G / B^{-} \times G / B$. On $a$ :

1) Les sous-variétés $\bar{V}_{G}^{X}$ et $Z$ s'intersectent proprement dans $X$.

2) Les composantes irréductibles de $\bar{V}_{G}^{X} \cap Z$ sont les adhérences dans $G / B^{-} \times G / B$ des orbites de $B \times H$

$$
B w B^{-} / B^{-} \times V_{G / B}^{\prime}
$$

telles que $w$ s'écrit $w(\gamma)$ pour un chemin $\gamma$ dans $\Gamma(G / H)$ qui monte de $V_{G} \grave{a}$ une orbite $V_{G / B}^{\prime}$ de $H$ dans $G / B$.

3) La multiplicité de l'intersection $\bar{V}_{G}^{X} \cap Z$ le long de $\overline{B w(\gamma) B^{-}} / B^{-} \times \bar{V}_{G / B}^{\prime}$ est $2^{D(\gamma)}$.

Sous certaines hypothèses, le théorème 4.13 donne une expression pour la classe $\left[\bar{V}_{G}^{X}\right]$ de cohomologie $B \times\{1\}$-équivariante (ou $T \times\{1\}$-équivariante) de $\bar{V}_{G}^{X}$ à coefficients rationnels. Ici, ceci nous donne le

ThÉorÈme D. - Dans $H_{B \times\{1\}}^{*}(X)$, on $a$ :

$$
\left[\bar{V}_{G}^{X}\right]=\sum_{w=w(\gamma)} 2^{D(\gamma)}\left[\overline{B w^{-1} B^{-}} \times\right]
$$

où la somme porte sur tous les chemins $\gamma$ issus de $V_{G}$.

Remarquons que la formule du théorème D est valable dans la cohomologie ordinaire $H^{*}(X)$ de $X$. En revanche, la démonstration faite en 4.5 utilise fortement la cohomologie équivariante.

Considérons un plongement lisse $G$-équivariant et toroïdal $Y$ de $G / H$ et $\mathcal{O}$ une orbite de $G$ dans $Y$. Soit $V$ une orbite de $B$ dans $G / H$. M. Brion demande dans [4], après la proposition 6 , si chaque composante irréductible de $\bar{V}^{Y} \cap \overline{\mathcal{O}}^{Y}$ contient des points lisses de $\bar{V}^{Y}$. Nous répondons à cette question par la négative dans la dernière partie.

En effet, nous considérons l'unique orbite fermée $V$ d'un sous-groupe de Borel $B$ dans l'espace homogène $\operatorname{PSL}(4) / \mathrm{PSO}(4)$. Soit $Y$ le plongement canonique de PSL(4)/PSO(4) et $Z$ l'unique orbite fermée de PSL(4) dans $Y$. Le dernier résultat (théorème 5.1 dans le corps du texte) est le

ThÉORÈme E. - Avec les notations ci-dessus, l'intersection $Z \cap \bar{V}^{Y}$ a une composante irréductible constituée de points singuliers de $\bar{V}^{Y}$.

La majeure partie de cet article (les théorèmes A, B, C et D) étudie les adhérences d'orbites de $B \times H$ dans les plongements de $G$. Ce travail s'inspire de celui de M. Brion [4] sur les adhérences d'orbites de $B$ dans les plongements

TOME $132-2004-\mathrm{N}^{\mathrm{O}} 4$ 
de $G / H$. Par exemple, le théorème B (ou le théorème 4.9 ci-dessous) est l'analogue de la proposition 6 de [4]. On peut remarquer que la description de la variété $S_{V, w(\gamma)}$ du théorème B est plus précise que celle de la variété $S_{Y, w}$ de M. Brion. Il est par exemple facile de déterminer le lieu non lisse (ou non normal) de $S_{V, w(\gamma)}$. Le théorème C (ou 4.10) est l'analogue du théorème 1 de [4]. Avec les notations du théorème $\mathrm{C}$, une différence notable est qu'ici tous les chemins dans $\Gamma(G / H)$ issus de $V_{G}$ interviennent dans la description de $\bar{V}_{G}^{X} \cap Z$; alors que, dans la situation étudiée par M. Brion seuls ceux arrivant à l'orbite ouverte comptent.

J'ai montré dans [13] que certains plongements $Y$ de $G / H$ s'obtiennent comme quotient géométriques par $H$ d'ouverts de plongements $X$ de $G$. Ainsi, sous certaines hypothèses, les adhérences d'orbites de $B$ dans $Y$ sont des quotients géométriques d'ouverts d'adhérences d'orbites de $B \times H$ dans $X$. Ceci est un lien explicite entre le travail de M. Brion [4] et cet article. Il est donc permis de penser que ce travail peut aider à mieux comprendre les adhérences d'orbites de $B$ dans des plongements de $G / H$. Ce point de vue m'a conduit à examiner l'exemple de la section 5 .

Remerciements. - Je tiens à remercier M. Brion pour ses encouragements et sa lecture attentive de versions préliminaires à cet article. Je voudrais également remercier S. Pin pour nos nombreuses et utiles discussions.

\section{Le graphe associé aux orbites de $H$ dans $\mathcal{B}$}

2.1. - Soient $G$ un groupe algébrique réductif connexe sur $\mathbb{C}$ et $\mathcal{B}$ la variété des drapeaux de $G$. Soit $H$ un sous-groupe algébrique de $G$ qui a une orbite dense dans $\mathcal{B}$; on dit alors que $H$ est sphérique. Nous nous intéressons à l'ensemble $\mathbf{H}(\mathcal{B})$ des orbites de $H$ dans $\mathcal{B}$. En fait, cet ensemble est fini (voir [1], [19] ou [9]).

Rappelons brièvement comment on construit un graphe dont les sommets sont les éléments de $\mathbf{H}(\mathcal{B})$. Le point de vue choisi ici est différent de celui de l'introduction ou de [4]. Il est cependant facile de voir que ces constructions sont équivalentes.

Considérons l'ensemble $\Delta$ des classes de conjugaison de sous-groupes paraboliques non résolubles minimaux de $G$. Si $\alpha$ appartient à $\Delta$, on note $\mathcal{P}_{\alpha}$ le $G$ espace homogène d'isotropie $\alpha$. Alors, il existe une unique application

$$
\phi_{\alpha}: \mathcal{B} \longrightarrow \mathcal{P}_{\alpha}
$$

qui est $G$-équivariante. Remarquons que les fibres de $\phi_{\alpha}$ sont isomorphes à $\mathbb{P}^{1}$.

Soit $V_{\alpha}$ une orbite de $H$ dans $\mathcal{P}_{\alpha}$ et $v$ un point de $V_{\alpha}$. Chaque orbite de $H$ dans $\phi_{\alpha}^{-1}\left(V_{\alpha}\right)$ intersecte $\phi_{\alpha}^{-1}(v)$ suivant une orbite du stabilisateur $H_{v}$ de $v$ dans $H$. Considérons le morphisme

$$
\theta: H_{v} \longrightarrow \operatorname{Aut}\left(\phi_{\alpha}^{-1}(v)\right) \simeq \operatorname{PSL}(2)
$$

BULlETIN DE LA SOCIÉTÉ MATHÉMATIQUE DE FRANCE 
induit par l'action de $H_{v}$ sur $\phi_{\alpha}^{-1}(v)$. Comme l'ensemble $\mathbf{H}(\mathcal{B})$ est fini, $H_{v}$ n'a qu'un nombre fini d'orbites dans $\phi_{\alpha}^{-1}(v)$. Autrement dit, l'image de $\theta$ est un sous-groupe sphérique de $\operatorname{Aut}\left(\phi_{\alpha}^{-1}(v)\right)$. Il y a quatre possibilités :

- Type $S L$ : $\theta$ est surjective.

Alors, $\phi_{\alpha}^{-1}\left(V_{\alpha}\right)$ est une orbite de $H$.

- Type $T: \operatorname{Im} \theta$ est un tore maximal de $\operatorname{Aut}\left(\phi_{\alpha}^{-1}(v)\right)$.

Alors, $H$ a trois orbites dans $\phi_{\alpha}^{-1}\left(V_{\alpha}\right)$ : une ouverte et deux de codimension 1.

- Type $N: \operatorname{Im} \theta$ est le normalisateur d'un tore maximal de $\operatorname{Aut}\left(\phi_{\alpha}^{-1}(v)\right)$.

Alors, $H$ a deux orbites dans $\phi_{\alpha}^{-1}\left(V_{\alpha}\right)$ : une ouverte et une de codimension 1.

- Type $U: \theta$ est non surjective et $\operatorname{Im} \theta$ contient un sous-groupe unipotent non trivial de $\operatorname{Aut}\left(\phi_{\alpha}^{-1}(v)\right)$.

Alors, $H$ a deux orbites dans $\phi_{\alpha}^{-1}\left(V_{\alpha}\right)$ : une ouverte et une de codimension 1.

Soit $V_{\mathcal{B}}$ une orbite de $H$ dans $\mathcal{B}$.

- On dit que $\alpha$ monte $V_{\mathcal{B}}$ si $V_{\mathcal{B}}$ est strictement inclus dans $\phi_{\alpha}^{-1}\left(\phi_{\alpha}\left(V_{\mathcal{B}}\right)\right)$; alors $V_{\mathcal{B}}$ est de codimension 1 dans $\phi_{\alpha}^{-1}\left(\phi_{\alpha}\left(V_{\mathcal{B}}\right)\right)$.

- Si $V_{\mathcal{B}}^{\prime}$ est l'orbite ouverte de $H$ dans $\phi_{\alpha}^{-1}\left(\phi_{\alpha}\left(V_{\mathcal{B}}\right)\right)$, on dit que $\alpha$ monte $V_{\mathcal{B}}$ $\operatorname{sur} V_{\mathcal{B}}^{\prime}$.

Si $\alpha$ monte $V_{\mathcal{B}}$, la restriction de $\phi_{\alpha}$ à $V_{\mathcal{B}}$ est fini. De plus, son degré $d\left(V_{\mathcal{B}}, \alpha\right)$ vaut 1 pour les types $T$ et $U$ et vaut 2 pour le type $N$.

2.2. On construit un graphe orienté $\Gamma(G / H)$ comme suit :

DÉfinition 2.1. - Les sommets de $\Gamma(G / H)$ sont les éléments de $\mathbf{H}(\mathcal{B})$. Le sommet $V_{\mathcal{B}}$ est l'origine d'une arête indexée par $\alpha$ et d'extrémité $V_{\mathcal{B}}^{\prime}$ si et seulement si $\alpha$ monte $V_{\mathcal{B}}$ sur $V_{\mathcal{B}}^{\prime}$. De plus, cette arête est simple (resp. double) si $d\left(V_{\mathcal{B}}, \alpha\right)$ vaut 1 (resp. 2$)$.

D'après ce qui précède, tout graphe $\Gamma(G / H)$ est construit à partir des quatre «briques élémentaires » de la figure 1. Les sommets de chacune de ces briques sont les orbites de $H$ dans la préimage d'une même orbite de $H$ dans $\mathcal{P}_{\alpha}$. Nous donnons dans la section 5 un exemple de graphe $\Gamma(G / H)$; M. Brion en donne plusieurs autres dans [4].

2.3. Notons $\mathcal{H}$ le $G$-espace homogène d'isotropie $H$. Fixons un sous-groupe de Borel $B$ de $G$ tel que $B H$ est dense dans $G$ (on dira que $B$ est opposé à $H$ ). Notons que $B$ est unique à conjugaison par un élément de $H$ près. Si $V_{\mathcal{B}}$ appartient à $\mathbf{H}(\mathcal{B})$, on pose :

$$
V_{\mathcal{H}}:=\left\{g H / H: g^{-1} B / B \in V_{\mathcal{B}}\right\} .
$$

Alors, $V_{\mathcal{H}}$ est une orbite de $B$ dans $\mathcal{H}$ et l'application $V_{\mathcal{B}} \mapsto V_{\mathcal{H}}$ est une bijection de $\mathbf{H}(\mathcal{B})$ sur l'ensemble $\mathbf{B}(\mathcal{H})$ des orbites de $B$ dans $\mathcal{H}$.

TOME $132-2004-\mathrm{N}^{\mathrm{O}} 4$ 


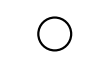

Type $S L$

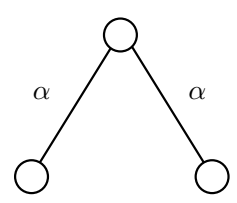

Type $T$

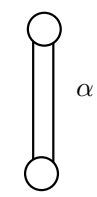

Type $N$

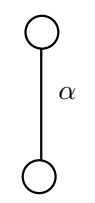

Type $U$

Figure 1. Briques élémentaires de $\Gamma(G / H)$.

2.4. Soit $T$ un tore maximal de $B$ et $W$ le groupe de Weyl de $T$. Tout $\alpha$ dans $\Delta$ a un unique représentant $P_{\alpha}$ contenant $B$. De plus, il existe un unique $s_{\alpha}$ dans $W$ tel que $B s_{\alpha} B$ soit dense dans $P_{\alpha}$; ce $s_{\alpha}$ est une réflexion simple de $W$. Enfin, l'application $\Delta \rightarrow W, \alpha \mapsto s_{\alpha}$ est une bijection de $\Delta$ sur l'ensemble des réflexions simples de $W$.

Soient $V_{\mathcal{H}}$ dans $\mathbf{B}(\mathcal{H})$ et $\alpha$ dans $\Delta$. Le groupe $B$ agit sur $P_{\alpha} \times V_{\mathcal{H}}$ par $b \cdot(p, v)=\left(p b^{-1}, b v\right)$ (avec des notations évidentes). Alors le quotient de $P_{\alpha} \times V_{\mathcal{H}}$ par $B$ existe et est noté $P_{\alpha} \times_{B} V_{\mathcal{H}}$ (voir l'annexe 6). On note $[p: v]$ la classe de $(p, v)$ dans $P_{\alpha} \times{ }_{B} V_{\mathcal{H}}$ si $p \in P_{\alpha}$ et $v \in V_{\mathcal{H}}$. Considérons l'application

$$
\pi_{\alpha}: P_{\alpha} \times_{B} V_{\mathcal{H}} \longrightarrow \mathcal{H}, \quad[p: v] \longmapsto p v .
$$

Soit $V_{\mathcal{H}}^{\prime}$ l'orbite ouverte de $B$ dans l'image de $\pi_{\alpha}$. Notons $V_{\mathcal{B}}$ et $V_{\mathcal{B}}^{\prime}$ les orbites de $H$ dans $\mathcal{B}$ correspondant à $V_{\mathcal{H}}$ et $V_{\mathcal{H}}^{\prime}$. On montre aisément que $\alpha$ monte $V_{\mathcal{B}}$ sur $V_{\mathcal{B}}^{\prime}$ si et seulement si $\pi_{\alpha}$ est finie sur son image. Dans ce cas, le degré de $\pi_{\alpha}$ est $d\left(V_{\mathcal{B}}, \alpha\right)$. Ceci nous conduit à poser la définition suivante :

DÉfinition 2.2. - Soient $V_{\mathcal{H}}, V_{\mathcal{H}}^{\prime}$ dans $\mathbf{B}(\mathcal{H})$ et $w$ dans $W$. On dit que $w$ monte $V_{\mathcal{H}}$ sur $V_{\mathcal{H}}^{\prime}$ si l'application (propre)

$$
\pi_{w}: \overline{B w B} \times_{B} \bar{V}_{\mathcal{H}} \longrightarrow \bar{V}_{\mathcal{H}}^{\prime}, \quad[p: v] \longmapsto p v .
$$

est surjective et génériquement finie. On note $d\left(V_{\mathcal{H}}, w\right)$ le degré de $\pi_{w}$.

\section{Orbites de $B \times H$ dans $G$}

3.1. On munit $G$ de l'action de $G \times G$ définie par $\left(g_{1}, g_{2}\right) \cdot g=g_{1} g g_{2}^{-1}$ pour tout $g, g_{1}$ et $g_{2}$ dans $G$. On considère la restriction de cette action à $B \times H$ et on note $\mathbf{B H}(G)$ l'ensemble des orbites de $B \times H$ dans $G$. Si $V_{\mathcal{B}} \in \mathbf{H}(\mathcal{B})$, on pose

$$
V_{G}=\left\{g \in G: g^{-1} B / B \in V_{\mathcal{B}}\right\} .
$$

Alors, $V_{\mathcal{B}} \mapsto V_{G}$ est une bijection de $\mathbf{H}(\mathcal{B})$ sur $\mathbf{B H}(G)$. 
3.2. Notons $\mathcal{V}$ l'ensemble des triplets $\left(V_{\mathcal{B}}, V_{\mathcal{H}}, V_{G}\right) \in \mathbf{H}(\mathcal{B}) \times \mathbf{B}(\mathcal{H}) \times \mathbf{B H}(G)$ dont les trois composantes coïncident via les bijections des paragraphes 2.3 et 3.1. Par exemple, le triplet $V^{0}:=(H \cdot B / B, B \cdot H / H, B H)$ est dans $\mathcal{V}$; on l'appelle l'orbite ouverte. Si $V$ est un élément de $\mathcal{V}$, alors $V_{\mathcal{B}}\left(\operatorname{resp}\right.$. $V_{\mathcal{H}}$ et $V_{G}$ ) désigne la projection de $V \operatorname{sur} \mathbf{H}(\mathcal{B})(\operatorname{resp} . \mathbf{B}(\mathcal{H})$ et $\mathbf{B H}(G))$. Soit $V$ et $V^{\prime}$ dans $\mathcal{V}$ et $w$ dans $W$. Nous dirons que $w$ monte $V$ sur $V^{\prime}$ si $w$ monte $V_{\mathcal{H}}$ sur $V_{\mathcal{H}}^{\prime}$. Dans ce cas on pose $d(V, w):=d\left(V_{\mathcal{H}}, w\right)$.

3.3. Si $w$ appartient à $W$, on pose $B_{w}:=B \cap w B w^{-1}$. Considérons l'application

$$
\eta: G \longrightarrow \mathcal{B}, \quad g \longmapsto g^{-1} B / B .
$$

Soit $V$ et $V^{\prime}$ dans $\mathcal{V}$ et $w$ dans $W$ qui monte $V$ sur $V^{\prime}$. Fixons un point $v$ dans $V_{\mathcal{B}}^{\prime}$. Notons $w V_{G}$ pour $(w, 1) . V_{G}$ et posons

$$
\Sigma_{V, w}:=w V_{G} \cap \eta^{-1}(v) .
$$

Soit $H_{v}$ le stabilisateur de $v$ dans $H$. Alors, $B_{w} \times H_{v}$ stabilise $\Sigma_{V, w}$. En fait, $B_{w} \times H_{v}$ agit transitivement sur $\Sigma_{V, w}$ et on a même le résultat plus précis suivant :

Proposition 3.1. - On a :

1) La variété $\Sigma_{V, w}$ possède $d(V, w)$ composantes irréductibles (ou connexes). Chacune est une orbite de $B_{w}$.

2) Le groupe $H_{v}$ agit transitivement sur l'ensemble des composantes irréductibles de $\Sigma_{V, w}$.

3) Le produit fibré $H \times_{H_{v}} \Sigma_{V, w}$ existe (voir l'annexe 6). De plus, l'application $H \times_{H_{v}} \Sigma_{V, w} \longrightarrow w V_{G}$ est une immersion ouverte.

Démonstration. - Il est clair que $B_{w} \times H_{v}$ stabilise $w V_{G}$ et $\eta^{-1}(v)$. Il stabilise alors $\Sigma_{V, w}$. Posons $\Omega=\eta^{-1}(H \cdot v) \cap w V_{G}$. Alors, $\Omega$ est ouvert dans $w V_{G}$ et la restriction de $\eta$ à $\Omega$ est un morphisme $H$-équivariant de $\Omega$ sur $H \cdot v$. De plus, la fibre au-dessus de $v$ est $\Sigma_{V, w}$. L'assertion 3) découle alors du lemme 6.1 en annexe.

Comme $\Omega$ est ouvert dans $w V_{G}, H \times_{H_{v}} \Sigma_{V, w}$ est irréductible. On en déduit l'assertion 2).

Considérons l'application naturelle $\pi_{w}: B w B \times{ }_{B} V_{\mathcal{H}} \rightarrow B w V_{\mathcal{H}}$. Soit $g_{v}$ dans $G$ tel que $\eta\left(g_{v}\right)=v$. Posons $y=g_{v} H / H \in V_{\mathcal{H}}^{\prime} \subset B w V_{\mathcal{H}}$. Alors, $\pi_{w}^{-1}(y)$ est formé de $d(V, w)$ points. Soit $\widehat{w}$ un représentant de $w$ dans $N(T)$. Considérons l'application

$$
\zeta: \Sigma_{V, w} \longrightarrow \pi_{w}^{-1}(y), \quad g \longmapsto\left[g_{v} g^{-1} \widehat{w}: \widehat{w}^{-1} g H / H\right] .
$$

Vérifions que $\zeta$ est bien définie. Comme $\Sigma_{V, w}$ est inclus dans $B g_{v}$, si $g$ appartient à $\Sigma_{V, w}$ alors $g_{v} g^{-1}$ appartient à $B$. Ainsi, $g_{v} g^{-1} \widehat{w} \in B w B$.

Par ailleurs, $\Sigma_{V, w}$ est inclus dans $w V_{G}$; donc $\widehat{w}^{-1} g \in V_{G}$. Alors, $\widehat{w}^{-1} g H / H$ appartient à $V_{\mathcal{H}}$. Ainsi, $\left[g_{v} g^{-1} \widehat{w}: \widehat{w}^{-1} g H / H\right]$ a bien un sens dans $B w B \times_{B} V_{\mathcal{H}}$.

TOME $132-2004-\mathrm{N}^{\mathrm{O}} 4$ 
Enfin, $\pi_{w}\left(\left[g_{v} g^{-1} \widehat{w}: \widehat{w}^{-1} g H / H\right]\right)=g_{v} g^{-1} \widehat{w} \widehat{w}^{-1} g H / H=y$. Ainsi, $\zeta$ est bien définie.

Montrons que $\zeta$ est surjective. Soient $b$ dans $B$ et $x$ dans $V_{\mathcal{H}}$ tels que $\pi_{w}([b \widehat{w}: x])=y$. Comme $\widehat{w} x=b^{-1} y, b^{-1} g_{v}$ appartient à $w V_{G}$ puis à $\Sigma_{V, w}$. Mais alors, $x=\widehat{w}^{-1} b^{-1} y$ implique $[b \widehat{w}: x]=\zeta\left(b^{-1} g_{v}\right)$ et la surjectivité de $\zeta$.

Montrons que les fibres de $\zeta$ sont des orbites de $B_{w}$. Soient $g_{1}$ et $g_{2}$ dans $\Sigma_{V, w}$. Si $\zeta\left(g_{1}\right)=\zeta\left(g_{2}\right)$, alors il existe $b$ dans $B$ tel que

$$
\begin{aligned}
g_{v} g_{1}^{-1} \widehat{w} b & =g_{v} g_{2}^{-1} \widehat{w} \\
b^{-1} \widehat{w}^{-1} g_{1} g_{v}^{-1} \cdot y & =\widehat{w}^{-1} g_{2} g_{v}^{-1} \cdot y .
\end{aligned}
$$

L'égalité (1) montre que $g_{1} g_{2}^{-1}=\widehat{w} b \widehat{w}^{-1}$. Par ailleurs, $\eta\left(g_{1}\right)=\eta\left(g_{2}\right)$. Ainsi, $g_{1} g_{2}^{-1}$ appartient à $B_{w}$. Réciproquement, si $g_{1} g_{2}^{-1}$ appartient à $B_{w}$ alors l'élément $b=\widehat{w}^{-1} g_{1} g_{2}^{-1} \widehat{w}$ de $B$ vérifie les égalités (1) et $(2)$.

On a montré que les fibres de $\zeta$ sont les orbites de $B_{w}$ dans $\Sigma_{V, w}$. Comme $B_{w}$ est connexe, ceci montre que chaque composante irréductible (ou connexe) de $\Sigma_{V, w}$ est une orbite de $B_{w}$. Le fait que $\Sigma_{V, w}$ possède $d(V, w)$ composantes irréductibles découle alors de la surjectivité de $\zeta$.

\section{Orbites de $B \times H$ dans les plongements de $G$}

\subsection{Préliminaire sur les plongements du groupe}

4.1.1. Soit $Y$ une variété normale munie d'une action algébrique de $G$. Si $Y$ est de plus munie d'une immersion ouverte et $G$-équivariante de $\mathcal{H}$ dans $Y$, on dit que $Y$ est un plongement de $\mathcal{H}$. Un plongement $Y$ de $\mathcal{H}$ est dit torö̈dal si tout diviseur irréductible stable par $B$ de $Y$ qui contient une orbite de $G$ est stable par $G$. Soit $\Delta_{Y}$ la réunion des diviseurs de $Y$ stables par $B$ et non par $G$. Soit $P_{\mathcal{H}}$ le stabilisateur dans $G$ de l'orbite ouverte de $B$ dans $\mathcal{H}$ et $P_{\mathcal{H}}^{u}$ son radical unipotent. Supposons que $Y$ est torö̈dal. Alors, il existe un sous-groupe de Levi $L_{\mathcal{H}}$ de $P_{\mathcal{H}}$, ne dépendant que de $\mathcal{H}$, et une sous-variété fermée $S$ de $Y-\Delta_{Y}$ stable par $L_{\mathcal{H}}$, tels que l'application

$$
P_{\mathcal{H}}^{u} \times S \longrightarrow Y-\Delta_{Y}, \quad(p, s) \longmapsto p s
$$

est un isomorphisme. De plus, le sous-groupe dérivé de $L_{\mathcal{H}}$ agit trivialement sur $S$, qui est donc une variété torique. Enfin, toute orbite de $G$ dans $Y$ rencontre $S$ suivant une unique orbite de $L_{\mathcal{H}}$.

Le groupe $G$ muni de l'action de $G \times G$ définie en 3.3 est un $G \times G$-espace homogène sphérique (et même symétrique). Les plongements de cet espace homogène seront simplement appelés plongements du groupe $G$. Chacun d'eux ne contient qu'un nombre fini d'orbites de $B \times B$ et donc de $G \times G$. 
4.1.2. Nous oublions le sous-groupe sphérique $H$ jusqu'à la fin de cette soussection pour nous concentrer sur les plongements torö̈daux du groupe $G$. Soit $X$ un tel plongement. Le premier résultat que nous rappelons décrit la structure locale d'un tel plongement.

Commençons par fixer quelques notations. Soient $B$ et $B^{-}$deux sous-groupes de Borel opposés de $G$. Posons $T=B \cap B^{-}$. Notons $U$ (resp. $U^{-}$) le radical unipotent de $B$ (resp. $B^{-}$).

Soit $\mathcal{O}$ une orbite de $G \times G$ dans $X$. Posons :

$$
\begin{aligned}
X_{\mathcal{O}} & :=\left\{x \in X: \overline{(G \times G) \cdot x}^{X} \supset \mathcal{O}\right\}, \\
X_{\mathcal{O}, B \times B^{-}} & :=\left\{x \in X: \overline{\left(B \times B^{-}\right) \cdot x} \text { X } \supset \mathcal{O}\right\} .
\end{aligned}
$$

Remarquons que $X_{\mathcal{O}, B \times B^{-}}$contient $B B^{-}$et donc $T$. Considérons l'adhérence $S_{\mathcal{O}}$ de $T$ dans $X_{\mathcal{O}, B \times B^{-}}$. En fait, avec les notations du paragraphe 4.1.1, on a $X_{\mathcal{O}, B \times B^{-}}=X_{\mathcal{O}}-\Delta_{X_{\mathcal{O}}}$ et $S_{\mathcal{O}}=S$ pour $Y=X_{\mathcal{O}}$. Il est montré dans [2] la Proposition 4.1. - On a :

1) $X_{\mathcal{O}, B \times B^{-}}$est un ouvert affine de $X$.

2) L'application

$$
U \times U^{-} \times S_{\mathcal{O}} \longrightarrow X_{\mathcal{O}, B \times B^{-}}, \quad\left(u, u^{-}, s\right) \longmapsto\left(u, u^{-}\right) . s
$$

est un isomorphisme $B \times B^{-}$-équivariant.

3) Pour toute orbite $\mathcal{O}^{\prime}$ de $G \times G$ dans $X_{\mathcal{O}}, \mathcal{O}^{\prime} \cap S_{\mathcal{O}}$ est une orbite de $T \times T$.

4.1.3. On va maintenant décrire les isotropies dans $G \times G$ des points de $S_{\mathcal{O}}$. On note $\mathcal{X}_{*}(T)$ le groupe constitué des sous-groupes à un paramètre de $T$. Si $\lambda \in \mathcal{X}_{*}(T)$, on pose

$$
P(\lambda):=\left\{g \in G: \lim _{t \rightarrow 0} \lambda(t) g \lambda\left(t^{-1}\right) \text { existe dans } G\right\} .
$$

D'après [10], $P(\lambda)$ est un sous-groupe parabolique de $G$ dont le radical unipotent est

$$
P^{u}(\lambda)=\left\{g \in G: \lim _{t \rightarrow 0} \lambda(t) g \lambda\left(t^{-1}\right)=1\right\} .
$$

De plus, $P(-\lambda)$ est opposé à $P(\lambda)$ et leur sous-groupe de Levi commun est le centralisateur $L(\lambda)$ de l'image de $\lambda$. Enfin, on pose

$$
\Delta L(\lambda)=\{(\ell, \ell) \in G \times G: \ell \in L(\lambda)\}
$$

et on note $C(\lambda)$ le centre connexe de $L(\lambda)$.

Remarquons que (d'après la proposition 4.1) $S_{\mathcal{O}}$ est un plongement affine et $T \times T$-équivariant de $T$ dont l'orbite fermée est $S_{\mathcal{O}} \cap \mathcal{O}$. Alors, il existe un sous-groupe à un paramètre $\lambda$ de $T$ tel que $z:=\lim _{t \rightarrow 0} \lambda(t)$ existe et appartient à $S_{\mathcal{O}} \cap \mathcal{O}$. De plus, $z$ ne dépend que de $T, B$ et $\mathcal{O}$; il est appelé le point base de $\mathcal{O}$.

Proposition 4.2. - On a :

1) $P(-\lambda)$ contient $B$,

TOME $132-2004-\mathrm{N}^{\mathrm{O}} 4$ 
2) l'isotropie de $z$ dans $G \times G$ est le produit semi-direct de $P^{u}(\lambda) \times P^{u}(-\lambda)$ et de $\Delta L(\lambda) \cdot(C(\lambda) \times\{1\})_{z}$.

En particulier, $P(-\lambda)$ ne dépend que de $\mathcal{O}$ et de $B$.

La proposition A1 de [3] implique la proposition précédente sous des hypothèses sensiblement plus fortes. Cependant, la démonstration de [3] s'applique sans changement ici.

\subsubsection{Posons}

$$
X_{\mathcal{O}, B \times G}:=(\{1\} \times G) \cdot X_{\mathcal{O}, B \times B^{-}} .
$$

D'après la proposition $4.1, X_{\mathcal{O}, B \times G}$ rencontre chaque orbite de $G \times G$ dans $X_{\mathcal{O}}$ suivant une orbite de $B \times G$. On a alors la

Proposition 4.3. - Il existe une application $B \times G$-équivariante

$$
\psi: X_{\mathcal{O}, B \times G} \longrightarrow \mathcal{B}=(B \times G) /(B \times B) .
$$

De plus, la fibre au-dessus de $(B / B, B / B)$ est $U \times S_{\mathcal{O}}$. Enfin, si $\mathcal{O}^{\prime}$ est une orbite de $G \times G$ dans $X_{\mathcal{O}}$, chaque fibre de $\psi$ intersecte $\mathcal{O}^{\prime}$ suivant une orbite de $B \times\{1\}$.

Remarque 4.4. - Comme l'application naturelle $G \rightarrow G / B$ est une fibration localement triviale, celle de la proposition l'est aussi.

Démonstration. — Rappelons que $\eta$ est l'application $\eta: G \rightarrow \mathcal{B}, g \mapsto g^{-1} B / B$. Il est clair que $\eta$ s'étend en une application rationnelle de $X$ sur $\mathcal{B}, B \times\{1\}$ invariante et $\{1\} \times G$-équivariante. De plus, d'après la proposition 4.1 , la restriction de $\eta$ à $X_{\mathcal{O}, B \times B^{-}}$est régulière. On a même le diagramme commutatif suivant :

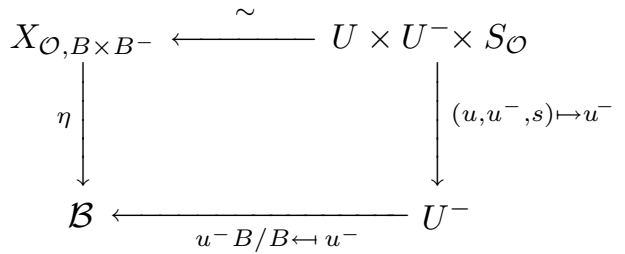

Alors, $\eta$ s'étend par $\{1\} \times G$-équivariance en une fibration $\psi$ localement triviale de fibre $U \times S_{\mathcal{O}}$.

Soit $\mathcal{O}^{\prime}$ une orbite de $G \times G$ dans $X_{\mathcal{O}}$. Alors, $\psi^{-1}(B / B) \cap \mathcal{O}^{\prime}$ qui vaut $\left((U \times\{1\}) \cdot S_{\mathcal{O}}\right) \cap \mathcal{O}^{\prime}$ ou encore $(U \times\{1\}) \cdot\left(S_{\mathcal{O}} \cap \mathcal{O}^{\prime}\right)$ est une orbite de $B \times\{1\}$ d'après la proposition 4.1. Soit $x \in \mathcal{B}$ et $g \in G$ tels que $g \cdot x=B / B$. Alors, $\psi^{-1}(x) \cap \mathcal{O}^{\prime}$ qui vaut $(1, g) \cdot\left(\psi^{-1}(B / B) \cap \mathcal{O}^{\prime}\right)$ est une orbite de $B \times\{1\}$. 


\subsection{Orbites de $B \times H$ dans une orbite de $G \times G$ dans $X$}

4.2.1. Considérons à nouveau un sous-groupe sphérique $H$ de $G$ et un sousgroupe de Borel $B$ opposé à $H$. Conservons les notations des propositions 4.1 et 4.2. Posons de plus

$$
P:=P(-\lambda), \quad Q:=Q(\lambda), \quad L:=L(\lambda), \quad C:=C(\lambda) .
$$

Nous allons décrire l'ensemble $\mathbf{B H}(\mathcal{O})$ des orbites de $B \times H$ dans $\mathcal{O}$. Nous nous intéressons d'abord à l'ensemble $\mathbf{B}(G / P)$ des orbites de $B$ dans $G / P$.

Remarquons que $T$ est un tore maximal de $B \cap L$. Notons $\Phi_{L}$ l'ensemble des racines de $(L, T), \Phi_{L}^{+}$celles de $(B \cap L, T)$ et $\Phi^{+}$celles de $(B, T)$. Posons

$$
W^{L}=\left\{w \in W: w \cdot \Phi_{L}^{+} \subseteq \Phi^{+}\right\} .
$$

La décomposition de Bruhat (voir [7] ou [17]) affirme alors que :

$$
G / P=\coprod_{w \in W^{L}} B w P / P .
$$

On identifie ainsi $W^{L}$ à $\mathbf{B}(G / P)$ par $w \mapsto B w P / P$.

Le groupe de Weyl $W$ est engendré par les réflexions simples associées aux racines simples de $(B, T)$. Si $w$ appartient à $W$, on note $\ell(w)$ et on appelle longueur de $w$ le nombre minimal de réflexions simples nécessaires pour écrire $w$ comme leur produit. L'interprétation géométrique de cette longueur est :

$$
\ell(w)=\operatorname{dim}(B w P / P), \quad \text { si } w \in W^{L} .
$$

Considérons l'application canonique $q_{P^{u}}: P \rightarrow P / P^{u}$. Remarquons que $q_{P^{u}}$ induit un isomorphisme de groupes de $L$ sur $P / P^{u}$. Nous utiliserons le

LEMME 4.5. - Soit $w$ dans $W^{L}$.

1) Le sous-groupe $w^{-1} B w$ contient $B \cap L$.

2) On $a: q_{P^{u}}\left(P \cap w^{-1} B w\right)=q_{P^{u}}(B \cap L)$.

Démonstration. - 1) Le tore $T$ est inclus dans $B \cap L$ et dans $w^{-1} B w$. Mais alors, comme $\Phi_{L}^{+} \subseteq w^{-1} \Phi^{+}$, l'algèbre de Lie de $B \cap L$ est incluse dans celle de $w^{-1} B w$. Or $B \cap L$ est connexe donc $w^{-1} B w$ contient $B \cap L$.

2) Le groupe $q_{P^{u}}\left(P \cap w^{-1} B w\right)$ est résoluble puisque $P \cap w^{-1} B w$ l'est. Or la première assertion montre que $q_{P^{u}}\left(P \cap w^{-1} B w\right)$ contient l'image par $q_{P^{u}}$ de $B \cap L$. Comme la restriction de $q_{P^{u}}$ à $L$ est un isomorphisme, $q_{P^{u}}(B \cap L)$ est un sous-groupe de Borel de $P / P^{u}$ et la deuxième assertion suit.

4.2.2. L'inclusion de $(G \times G)_{z}$ dans $P \times G$ induit un morphisme $G \times G$ équivariant

$$
p: \mathcal{O}=G \times G /(G \times G)_{z} \longrightarrow G / P=(G \times G) /(P \times G) .
$$

On vérifie aisément que l'application

$$
q: p^{-1}(P / P) \longrightarrow \mathcal{B}, \quad\left(\ell p^{u}, g\right) \cdot z \longmapsto g \ell^{-1} B / B,
$$

TOME $132-2004-\mathrm{N}^{\mathrm{O}} 4$ 
(où $p^{u} \in P^{u}, \ell \in L$ et $g \in G$ ) définit un morphisme $\{1\} \times G$-équivariant de $p^{-1}(P / P)$ sur $\mathcal{B}$.

Proposition 4.6. - On a :

1) Soit $V_{\mathcal{O}} \in \mathbf{B H}(\mathcal{O})$. Alors, il existe un unique couple $\left(w, V^{\prime}\right) \in W^{L} \times \mathcal{V}$ tel que

$$
p\left(V_{\mathcal{O}}\right)=B w P / P \text { et } w^{-1} V_{\mathcal{O}} \cap p^{-1}(P / P)=q^{-1}\left(V_{\mathcal{B}}^{\prime}\right) .
$$

2) L'application $\mathbf{B H}(\mathcal{O}) \rightarrow W^{L} \times \mathcal{V}, V_{\mathcal{O}} \mapsto\left(w, V^{\prime}\right)$ définie par l'assertion 1$)$ est une bijection. On notera $\mathbf{B H}_{\mathcal{O}}\left(w, V^{\prime}\right)$ la préimage de $\left(w, V^{\prime}\right)$.

Démonstration. - Soit $V_{\mathcal{O}} \in \mathbf{B H}(\mathcal{O})$. L'image de $V_{\mathcal{O}}$ par $p$ est une orbite de $B$ dans $G / P$. Ceci implique l'existence et l'unicité de $w \in W^{L}$ tel que $p\left(V_{\mathcal{O}}\right)=B w P / P$.

De plus, $p^{-1}(P / P) \cap w^{-1} V_{\mathcal{O}}$ est une orbite de $\left(w^{-1} B w \cap P\right) \times H$. Mais alors, l'assertion 2) du lemme 4.5 implique que $p^{-1}(P / P) \cap w^{-1} V_{\mathcal{O}}$ est une orbite de $(B \cap L) \times H$ dans $p^{-1}(P / P) \simeq(P \times G) /\left(P^{u} \times Q^{u}\right) \cdot \Delta L \cdot C_{z}$. L'assertion 1$)$ suit aisément.

L'assertion 2) est alors banale.

Corollaire 4.7. - Soit $X$ un plongement du groupe $G$. Alors, $B \times H$ n'a qu'un nombre fini d'orbites dans $X$.

Démonstration. - Il est montré dans [2] l'existence d'un plongement toroïdal $\widetilde{X}$ de $G$ et d'un morphisme surjectif et $G \times G$-équivariant de $\widetilde{X}$ sur $X$. Donc, il suffit de montrer le corollaire pour $\widetilde{X}$. Or, la proposition 4.6 s'applique à chaque orbite de $G \times G$ dans $\widetilde{X}$ : le corollaire est démontré.

\subsection{Description d'ouverts de $\bar{V}_{G}^{X}$}

4.3.1. - On se donne deux éléments $V, V^{\prime}$ de $\mathcal{V}$ et $w \in W$ qui monte $V$ sur $V^{\prime}$. Rappelons que $\bar{V}_{G}^{X}$ désigne l'adhérence de $V_{G}$ dans $X$.

Reprenons les notations du paragraphe 3.3 ; en particulier, $v$ est un point de $V_{\mathcal{B}}^{\prime}$. Considérons l'adhérence $\overline{\Sigma_{V, w, \mathcal{O}}}$ de $\Sigma_{V, w}$ dans $X_{\mathcal{O}, B \times G}$. D'après la proposition $3.1, H_{v}$ agit sur $\overline{\Sigma_{V, w, \mathcal{O}}}$. Alors, on a le

LEMME 4.8. - Reprenons les notations de la proposition 4.3. Alors, le produit fibré $H \times_{H_{v}} \overline{\Sigma_{V, w, \mathcal{O}}}$ existe. De plus, l'application naturelle $H \times_{H_{v}} \overline{\bar{\Sigma}_{V, w, \mathcal{O}}} \rightarrow w \bar{V}_{G}^{X}$ est une immersion ouverte.

Démonstration. - Le lemme 6.1 de l'annexe montre ici que $w \bar{V}_{G}^{X} \cap \psi^{-1}\left(V_{\mathcal{B}}^{\prime}\right)$ est isomorphe à $H \times_{H_{v}}\left(w \bar{V}_{G}^{X} \cap \psi^{-1}(v)\right)$. Il reste à montrer l'égalité

$$
w \bar{V}_{G}^{X} \cap \psi^{-1}(v)=\overline{\Sigma_{V, w, \mathcal{O}}} .
$$

L'inclusion de $\overline{\Sigma_{V, w, \mathcal{O}}}$ dans le membre de gauche de l'égalité est évidente. Soit $C$ une composante irréductible de $w \bar{V}_{G}^{X} \cap \psi^{-1}(v)$. Alors, $(\{1\} \times H) \cdot C$ est une 
composante irréductible de $w \bar{V}_{G}^{X} \cap \psi^{-1}\left(V_{\mathcal{B}}^{\prime}\right)$. Comme $\psi\left(w \bar{V}_{G}^{X}\right)$ est inclus dans l'adhérence de $V_{\mathcal{B}}^{\prime}, \psi^{-1}\left(V_{\mathcal{B}}^{\prime}\right) \cap w \bar{V}_{G}^{X}$ est ouvert dans $w \bar{V}_{G}^{X}$. En particulier, toutes les composantes irréductibles de $\psi^{-1}\left(V_{\mathcal{B}}^{\prime}\right) \cap w \bar{V}_{G}^{X}$ rencontrent l'orbite ouverte $G$. Donc $(\{1\} \times H) \cdot C$ rencontre $G$. Mais alors, $C$ rencontre $G \cap \psi^{-1}(v)$. On en déduit que $C$ est inclus dans $\overline{\Sigma_{V, w, \mathcal{O}}}$.

4.3.2. - On veut maintenant décrire plus en détail la géométrie de $\overline{\Sigma_{V, w, \mathcal{O}}}$. Rappelons que $U_{w}$ désigne le groupe $U \cap w U w^{-1}$.

Soit $g$ dans $G$ tel que $g \cdot B / B=v$. Rappelons que la multiplication induit un isomorphisme $T$-équivariant du produit $U_{w} \times\left(U \cap w U^{-} w^{-1}\right)$ sur $U$. Alors, la proposition 4.3 montre que l'application

$$
\Theta: U_{w} \times\left(U \cap w U^{-} w^{-1}\right) \times S_{\mathcal{O}} \longrightarrow \psi^{-1}(v), \quad\left(u_{1}, u_{2}, s\right) \longmapsto\left(u_{1} u_{2}, g\right) \cdot s,
$$

est un isomorphisme $T \times\{1\}$-équivariant. Posons

$$
S_{V, w, \mathcal{O}}:=\overline{\Sigma_{V, w, \mathcal{O}}} \cap\left(\left(U \cap w U^{-} w^{-1}, g\right) \cdot S_{\mathcal{O}}\right) .
$$

Il est clair que l'action de $B_{w}$ sur $\Sigma_{V, w}$ induit une action de $T$ sur $S_{V, w, \mathcal{O}}$. Notons $\phi_{V, w}: \psi^{-1}(v) \rightarrow S_{\mathcal{O}}$ l'application $T$-équivariante définie par le diagramme commutatif suivant :

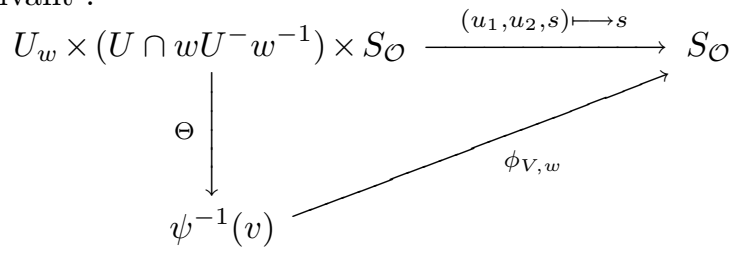

THÉORÈme 4.9. - Avec les notations ci-dessus, on a :

1) $\overline{\Sigma_{V, w, \mathcal{O}}}$ est isomorphe comme $B_{w}$-variété à $U_{w} \times S_{V, w, \mathcal{O}}$. En particulier, $S_{V, w, \mathcal{O}}$ possède $d(V, w)$ composantes irréductibles.

2) Si $w^{-1}$ appartient à $W^{L}$ (avec les notations du paragraphe 4.2.1), alors $S_{V, w, \mathcal{O}} \cap \mathcal{O}=S_{\mathcal{O}} \cap \mathcal{O}$. En particulier, $S_{V, w, \mathcal{O}} \cap \mathcal{O}$ est irréductible.

3) La restriction de $\phi_{V, w}$ à toute composante irréductible de $S_{V, w, \mathcal{O}}$ est un isomorphisme T-équivariant sur $S_{\mathcal{O}}$.

Démonstration. - La première assertion résulte immédiatement de l'invariance par $B_{w}$ de $\Sigma_{V, w}$.

Soit $S_{V, w, \mathcal{O}}^{1}$ une composante irréductible de $S_{V, w, \mathcal{O}}$. Toute composante irréductible de $\overline{\Sigma_{V, w, \mathcal{O}}}$ est l'adhérence d'une orbite de $B_{w}$ dans $G$. Mais alors, l'assertion 1) montre que $S_{V, w, \mathcal{O}}^{1}$ est l'adhérence dans $S_{V, w, \mathcal{O}}$ d'une orbite de $T$ dans $G$. On en déduit qu'il existe $u$ dans $U \cap w U^{-} w^{-1}$ tel que $(T \times\{1\}) \cdot(u, g)$ soit dense dans $S_{V, w, \mathcal{O}}^{1}$ (où $g$ est l'élément de $G$ utilisé dans la définition de $\Theta$ ). Fixons un tel $u$.

L'intersection $S_{V, w, \mathcal{O}}^{1} \cap \mathcal{O}$ est incluse dans $U_{w} \cdot\left(S_{\mathcal{O}} \cap \mathcal{O}\right)$. On en déduit que tout point de $S_{V, w, \mathcal{O}}^{1} \cap \mathcal{O}$ est de la forme $\lim _{t \rightarrow 0} \lambda(t) \cdot u$, pour un sous-groupe à

TOME $132-2004-\mathrm{N}^{\mathrm{O}} 4$ 
un paramètre $\lambda$ de $T$ tel que $\lim _{t \rightarrow 0} \lambda(t)$ existe et appartient à $S_{\mathcal{O}} \cap \mathcal{O}$. Alors, $L$ est le centralisateur de l'image de $\lambda$ (voir la proposition 4.2).

Supposons que $w^{-1}$ appartient à $W^{L}$. Alors, $\Phi_{L}^{+}$est inclus dans $w \cdot \Phi^{+}$. Donc, si $\alpha$ appartient à $\Phi_{L}^{+}$alors $w^{-1} \alpha$ n'appartient pas à $\Phi^{-}$. Ainsi, $\Phi^{+} \cap w \Phi^{-}$est inclus dans $\Phi^{+}$et ne rencontre pas $\Phi_{L}^{+}$. On en déduit que $U_{w}$ est inclus dans le radical unipotent $Q^{u}(\lambda)$ de $Q(\lambda)$. Or on a

$$
Q^{u}(\lambda)=\left\{g \in G: \lim _{t \rightarrow 0} \lambda(t) g \lambda\left(t^{-1}\right)=1\right\} .
$$

On en déduit que $\lim _{t \rightarrow 0} \lambda(t) \cdot u=\lim _{t \rightarrow 0} \lambda(t)$ appartient à $S_{\mathcal{O}} \cap \mathcal{O}$. L'assertion 2) est démontrée.

D'après la théorie des plongements des espaces homogènes sphériques (voir [8] ou [2]), il existe un plongement toroïdal $X^{\prime}$ de $G$ contenant $X_{\mathcal{O}}$ et dont l'unique orbite fermée $Z$ est projective. Il suffit alors de montrer l'assertion 3) lorsque $\mathcal{O}=Z$ est projective.

Considérons à nouveau une composante irréductible $S_{V, w, \mathcal{O}}^{1}$ de $S_{V, w, \mathcal{O}}$ et la restriction $\bar{\phi}_{V, w}: S_{V, w, \mathcal{O}}^{1} \rightarrow S_{\mathcal{O}}$ de $\phi_{V, w}$. L'application $\bar{\phi}_{V, w}$ est $T$-équivariante et dominante. De plus, comme $S_{V, w, \mathcal{O}}^{1}$ rencontre $G, S_{V, w, \mathcal{O}}^{1}$ contient un ouvert isomorphe à $T$ et $\bar{\phi}_{V, w}$ est birationnelle.

Considérons l'unique point $z_{0}$ de $S_{\mathcal{O}} \cap Z$. Alors, $\bar{\phi}_{V, w}^{-1}\left(z_{0}\right)$ est inclus dans $S_{V, w, \mathcal{O}}^{1} \cap Z$. Mais alors, l'assertion 2) montre que $\bar{\phi}_{V, w}^{-1}\left(z_{0}\right)=\left\{z_{0}\right\}$. On en déduit que $\bar{\phi}_{V, w}: S_{V, w, \mathcal{O}}^{1} \rightarrow S_{\mathcal{O}}$ est un isomorphisme.

\subsection{Intersection de $\bar{V}_{G}^{X}$ et d'une orbite de $G \times G$ dans $X$}

On conserve les notations de la sous-section précédente. On note $\overline{\mathcal{O}} X$ l'adhérence de $\mathcal{O}$ dans $X$. Dans cette sous-section, nous déterminons l'intersection $\overline{\mathcal{O}}^{X} \cap \bar{V}_{G}^{X}$. Nous utilisons la notation $\mathbf{B H}_{\mathcal{O}}\left(w, V^{\prime}\right)$ de la proposition 4.6.

ThÉORÈme 4.10. - L'intersection $\overline{\mathcal{O}}^{X} \cap \bar{V}_{G}^{X}$ est propre (i.e. sa codimension dans $X$ est la somme de celles de $\mathcal{O}$ et de $\left.V_{G}\right)$. De plus, on a

$$
\overline{\mathcal{O}}^{X} \cap \bar{V}_{G}^{X}=\bigcup \overline{\mathbf{B H}}_{\mathcal{O}\left(w, V^{\prime}\right)^{X}},
$$

où la réunion porte sur les couples $\left(w, V^{\prime}\right) \in W^{L} \times \mathcal{V}$ tels que $w^{-1}$ monte $V$ sur $V^{\prime}$. Si de plus $X$ est lisse, la multiplicité d'intersection de $\overline{\mathcal{O}}^{X} \cap \bar{V}^{X}$ le long

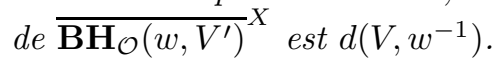

Démonstration. - Soit $C$ une composante irréductible de $\bar{V}_{G}^{X} \cap \overline{\mathcal{O}}^{X}$ qui rencontre $\mathcal{O}$. Comme $C$ est stable par $B \times H$, la proposition 4.6 montre qu'il existe $w$ dans $W^{L}$ et $V^{\prime}$ dans $\mathcal{V}$ tels que $C$ soit l'adhérence de $\mathbf{B H}_{\mathcal{O}}\left(w, V^{\prime}\right)$ dans $X$. 
Considérons la fibration $\psi: X_{\mathcal{O}, B \times G} \rightarrow \mathcal{B}$ définie par la proposition 4.3. Il est clair que $\psi\left(w^{-1} \bar{V}_{G}^{X}\right)$ est inclus dans $\overline{V_{G}^{-1} w B / B}$. Mais alors, $V_{\mathcal{B}}^{\prime}$ est inclus dans $\overline{V_{G}^{-1} w B / B}$.

Par ailleurs, la codimension de $C$ dans $\mathcal{O}$ est égale à la somme de la longueur de $w$ et de la codimension de $V_{\mathcal{B}}^{\prime}$ dans $\mathcal{B}$. Comme $C$ est une composante irréductible de $\bar{V}_{G}^{X} \cap \overline{\mathcal{O}}^{X}$, cette codimension est inférieure ou égale à celle de $V_{G}$ dans $G$, c'est-à-dire celle de $V_{\mathcal{B}}$ dans $\mathcal{B}$. On en déduit que $V_{\mathcal{B}}^{\prime}$ est l'orbite ouverte de $H$ dans $V_{G}^{-1} w B / B$ et que $w^{-1}$ monte $V$ sur $V^{\prime}$. De plus, on a :

$$
\operatorname{dim}\left(\overline{\mathcal{O}}^{X}\right)-\operatorname{dim}(C)=\operatorname{dim}(X)-\operatorname{dim}\left(\bar{V}_{G}^{X}\right)
$$

Soit $C^{\prime}$ une composante irréductible quelconque de $\bar{V}_{G}^{X} \cap \overline{\mathcal{O}}^{X}$. Alors, il existe une orbite $\mathcal{O}^{\prime}$ de $G \times G$ dans $\overline{\mathcal{O}}^{X}$ telle que $C^{\prime} \cap \mathcal{O}^{\prime}$ soit un ouvert non vide de $C^{\prime}$. L'égalité (3) appliquée à $\mathcal{O}^{\prime}$ et $C^{\prime}$ montre que $\mathcal{O}=\mathcal{O}^{\prime}$.

Ainsi, on a montré que l'intersection $\bar{V}_{G}^{X} \cap \overline{\mathcal{O}} X$ est propre et que toute composante irréductible de cette intersection est l'adhérence dans $X$ de $\mathbf{B H}_{\mathcal{O}}\left(w, V^{\prime}\right)$ pour un élément $w$ dans $W^{L}$ qui monte $V$ sur $V^{\prime}$.

Réciproquement, soit $w \in W^{L}$ et $V^{\prime}$ dans $\mathcal{V}$ tels que $w$ monte $V$ sur $V^{\prime}$. Notons $\mathcal{O}_{B \times G}$ l'orbite ouverte de $B \times G$ dans $\mathcal{O}$. Considérons l'application $p: \mathcal{O} \rightarrow G / P$ définie au paragraphe 4.2.2. Montrons que $p\left(\mathcal{O}_{B \times G} \cap w^{-1} \bar{V}_{G}^{X}\right)$ et $w^{-1} B w P / P$ ont la même adhérence dans $G / P$.

Comme $(\{1\} \times H) \cdot \Sigma_{V, w^{-1}}$ est ouvert dans $w^{-1} V_{G}$ et $p$ est $\{1\} \times G$-invariante, les adhérences de $p\left(w^{-1} \bar{V}_{G}^{X} \cap \mathcal{O}_{B \times G}\right)$ et de $p\left(\overline{\Sigma_{V, w^{-1}, \mathcal{O}}} \cap \mathcal{O}_{B \times G}\right)$ dans $G / P$ coïncident. Or, $p\left(\overline{\Sigma_{V, w^{-1}, \mathcal{O}}} \cap \mathcal{O}_{B \times G}\right)$ est égal à $\left(U \cap w^{-1} U w\right) \cdot p\left(S_{V, w^{-1}, \mathcal{O}} \cap \mathcal{O}_{B \times G}\right)$ qui est égal à $\left(U \cap w^{-1} U w\right) \cdot p\left(S_{\mathcal{O}} \cap \mathcal{O}_{B \times G}\right)$ d'après l'assertion 3$)$ du théorème 4.9. On en déduit que

$$
\overline{p\left(w^{-1} \bar{V}_{G}^{X} \cap \mathcal{O}_{B \times G}\right)}=w^{-1} \overline{B w P / P} .
$$

La proposition 4.6 montre alors qu'il existe une composante irréductible $C$ de $\bar{V}_{G}^{X} \cap \overline{\mathcal{O}}^{X}$ de la forme $\overline{\mathbf{B} \mathbf{H}_{\mathcal{O}}\left(w, V^{\prime \prime}\right)^{X}}$, pour $V^{\prime \prime}$ dans $\mathcal{V}$. Mais alors, le début de la démonstration montre que $V^{\prime \prime}=V^{\prime}$. Ainsi, $\overline{\mathbf{B H}_{\mathcal{O}}\left(w, V^{\prime}\right)^{X}}$ est une composante irréductible de $\bar{V}_{G}^{X} \cap \overline{\mathcal{O}}^{X}$.

De plus, au voisinage de $\mathbf{B H}_{\mathcal{O}}\left(w, V^{\prime}\right)$, l'application $\psi$ induit un isomorphisme d'un ouvert de $w^{-1} \bar{V}_{G}^{X}$ sur un produit fibré dont la fibre est $\overline{\Sigma_{V, w^{-1}, \mathcal{O}}}$. Mais alors, la multiplicité de $\bar{V}_{G}^{X} \cap \overline{\mathcal{O}}^{X}$ le long de $\overline{\mathbf{B H}}_{\mathcal{O}}\left(w, V^{\prime}\right)^{X}$ est égale à celle de $\overline{\Sigma_{V, w^{-1}, \mathcal{O}}} \cap \overline{\mathcal{O}}^{X}$. D'après le théorème 4.9 (assertions 2 et 3 ), cette multiplicité est celle de $S_{V, w^{-1}, \mathcal{O}} \cap \overline{\mathcal{O}}^{X}$ le long de $S_{\mathcal{O}} \cap \overline{\mathcal{O}}^{X}$. Si de plus $X$ est lisse, la proposition 4.1 montre que $S_{\mathcal{O}}$ est lisse. Mais alors, le théorème 4.9 montre que chacune des $d\left(V, w^{-1}\right)$ composantes irréductibles de $S_{V, w^{-1}, \mathcal{O}}$ est lisse. On en déduit que la multiplicité de $S_{V, w^{-1}, \mathcal{O}} \cap \overline{\mathcal{O}}^{X}$ le long de $S_{\mathcal{O}} \cap \overline{\mathcal{O}}^{X}$ est $d\left(V, w^{-1}\right)$ puis le théorème. 
Corollaire 4.11. - Supposons que $X$ est lisse et torö̈dal. Soit $C$ une composante irréductible de $\bar{V}_{G}^{X} \cap \overline{\mathcal{O}}^{X}$. Alors, se valent :

1) L'ouvert des points lisses de $\bar{V}_{G}^{X}$ rencontre $C$.

2) L'intersection $\bar{V}_{G}^{X} \cap \overline{\mathcal{O}}^{X}$ est transverse le long de $C$.

Démonstration. - Il suffit de montrer que si la multiplicité de l'intersection $\bar{V}_{G}^{X} \cap \overline{\mathcal{O}}^{X}$ le long de $C$ est supérieure ou égale à 2, alors $C$ est constitué de points singuliers de $\bar{V}_{G}^{X}$. Soit $w$ dans $W$ et $V^{\prime}$ dans $\mathcal{V}$ tels que l'orbite $\mathbf{B H}_{\mathcal{O}}\left(w, V^{\prime}\right)$ est ouverte dans $C$. Alors, la variété $\overline{\Sigma_{V, w, \mathcal{O}}}$ a au moins deux composantes irréductibles qui se rencontrent dans $C$. Mais alors, le corollaire découle du lemme 4.8 .

\subsection{Classe de cohomologie $B \times\{1\}$-équivariante de $\bar{V}_{G}^{X}$}

4.5.1. - Dans cette sous-section, nous fixons un plongement $X$ toroïdal complet et lisse de $G$. Rappelons que $B$ est un sous-groupe de Borel de $G$ opposé à $H$, que $B^{-}$est un sous-groupe de Borel de $G$ opposé à $B$, que $T$ égale $B \cap B^{-}$ et enfin que $V$ appartient à $\mathcal{V}$. Le théorème 4.13 ci-dessous donne une expression pour la classe de cohomologie $B \times\{1\}$-équivariante de $\bar{V}_{G}^{X}$.

Si $Y$ est une variété munie d'une action de $B$, on note $\mathrm{H}_{B}^{*}(Y)\left(\operatorname{resp} . \mathrm{H}_{T}^{*}(Y)\right)$ l'algèbre de cohomologie $B$-équivariante (resp. $T$-équivariante) de $Y$ à coefficients rationnels (voir [6] pour une définition). Comme l'application $B \rightarrow B / T$ est une fibration triviale dont la fibre est un espace affine, $\mathrm{H}_{B}^{*}(Y)$ et $\mathrm{H}_{T}^{*}(Y)$ sont canoniquement isomorphes. Dans la suite de cette section nous travaillerons avec la cohomologie $T$-équivariante.

La proposition 4.2 montre que les orbites fermées de $G \times G$ dans $X$ sont isomorphes à $\mathcal{B} \times \mathcal{B}$. Elle implique aussi que les points fixes de $T \times\{1\}$ dans $X$ appartiennent tous à des orbites fermées de $G \times G$ dans $X$. Ces deux propriétés de $X$ et le théorème de localisation en cohomologie $T$-équivariante (voir [6]) sont les ingrédients essentiels de la preuve du théorème 4.13 ci-dessous.

D'après la formule de Künneth, on a

$$
\mathrm{H}_{T \times\{1\}}^{*}(\mathcal{B} \times \mathcal{B})=\mathrm{H}_{T}^{*}(\mathcal{B}) \otimes \mathrm{H}^{*}(\mathcal{B}) .
$$

Nous utiliserons également le fait que $\mathrm{H}_{T}^{*}(\mathcal{B})$ est un $\mathrm{H}_{T}^{*}(\mathrm{pt})$-module libre (voir [6]).

Rappelons que $V^{0}=(H \cdot B / B, B \cdot H / H, B H) \in \mathcal{V}$ désigne l'orbite ouverte. 4.5.2. - Avec les notations ci-dessus, on a le

Lemme 4.12. - Soit $Z$ une orbite fermée de $G \times G$ dans $X$. Posons

$$
c=\sum_{\substack{w \in W \\ w \text { monte } V \text { sur } V^{0}}} d(V, w)\left[\overline{B w^{-1} B^{-}} X\right],
$$

dans $\mathrm{H}_{T \times\{1\}}^{*}(X)$. Alors, dans $\mathrm{H}_{T \times\{1\}}^{*}(X)$, on $a\left[\bar{V}_{G}^{X}\right] \cup[Z]=c \cup[Z]$. 
Démonstration. - Avec les notations de la proposition 4.1, $S_{Z} \cap Z$ est réduit à un point $z$ pour $\mathcal{O}=Z$. Alors, le couple $(Z, z)$ s'identifie à $\left(\mathcal{B} \times \mathcal{B},\left(B^{-} / B^{-}, B / B\right)\right)$. Avec cette identification, le théorème 4.10 montre que dans $\mathrm{H}_{T \times\{1\}}^{*}(X)$, on a

$$
\left[\bar{V}_{G}^{X}\right] \cup[Z]=\sum_{\substack{w \in W \\ w \text { monte } V \operatorname{sur} V^{\prime}}} d(V, w)\left[\overline{B w^{-1} B^{-}} / B^{-} \times V_{\mathcal{B}}^{\prime}\right] .
$$

Or, d'après [3], dans $\mathrm{H}^{*}(\mathcal{B})$ on a

$$
\left[V_{\mathcal{B}}^{\prime}\right]=\sum_{\substack{w \in W \\ w \operatorname{monte} V \operatorname{sur} V^{0}}} d\left(V^{\prime}, \tau\right)\left[\overline{B^{-} \tau B} / B\right]
$$

Alors, on obtient

$$
\left[\bar{V}_{G}^{X}\right] \cup[Z]=\sum d(V, w) d\left(V^{\prime}, \tau\right)\left[\overline{B w^{-1} B^{-}} / B^{-} \times \overline{B^{-} \tau B} / B\right],
$$

où la somme porte sur les couples $(w, \tau) \in W \times W$ tels que $w$ monte $V$ sur $V^{\prime}$ et $\tau$ monte $V^{\prime}$ sur $V^{0}$.

On associe à tout couple $(w, \tau)$ apparaissant dans cette somme le couple $\left(w_{1}, \tau_{1}\right) \in W \times W$ défini par

$$
w_{1}=\tau w, \tau_{1}=w, \quad w=\tau_{1}, \tau=w_{1} \tau_{1}^{-1} .
$$

Alors, $w_{1}$ monte $V$ sur $V^{0}$ et $\ell\left(\tau_{1}\right)+\ell\left(w_{1} \tau_{1}^{-1}\right)=\ell\left(w_{1}\right)$. Réciproquement, à tout couple $\left(w_{1}, \tau_{1}\right)$ satisfaisant ces deux conditions on peut associer par les formules (5) un couple $(w, \tau)$ apparaissant dans la somme (4). De plus, la condition « $\ell\left(\tau_{1}\right)+\ell\left(w_{1} \tau_{1}^{-1}\right)=\ell\left(w_{1}\right) »$ est équivalente à « $\tau_{1}$ monte $B w_{1}^{-1} B^{-} / B^{-}$ dans $\Gamma\left(G / B^{-}\right) »$. On en déduit que

$$
\left[\bar{V}_{G}^{X}\right] \cup[Z]=\sum_{\substack{w_{1} \text { monte } V \text { sur } V^{0} \\ \tau_{1} \text { monte } B w_{1}^{-1} B^{-} / B^{-}}} d\left(V, w_{1}\right)\left[\overline{B \tau_{1}^{-1} B^{-}} / B^{-} \times \overline{B^{-} w_{1} \tau_{1}^{-1} B} / B\right] .
$$

Par ailleurs, on a :

$$
c \cup[Z]=\sum_{w \text { monte } V \text { sur } V^{0}} d(V, w)\left[\overline{B w^{-1} B^{-}}\right] \cup[Z] .
$$

On applique alors le théorème 4.10 avec $H=B^{-}$à chaque $\left[\overline{B w^{-1} B^{-}}\right] \cup[Z]$. On obtient :

$$
c \cup[Z]=\sum_{\substack{w \text { monte } V \text { sur } V^{0} \\ \tau \text { monte } \overline{B w^{-1} B^{-}} / B^{-}}} d(V, w)\left[\overline{B \tau^{-1} B^{-}} / B^{-} \times \overline{B^{-} w \tau^{-1} B} / B\right] .
$$

Le lemme est démontré.

TOME $132-2004-\mathrm{N}^{\mathrm{O}} 4$ 
4.5.3. Au cours de la preuve du lemme 4.12, nous avons utilisé une expression de la classe de $\left[V_{\mathcal{B}}\right]$ dans $\mathrm{H}^{*}(\mathcal{B})$ démontrée dans [3]. Cette dernière nous dit que dans $\mathrm{H}_{T \times\{1\}}^{*}(G) \simeq \mathrm{H}^{*}(G / T) \simeq \mathrm{H}^{*}(\mathcal{B})$, on a :

$$
\left[\bar{V}_{G}^{G}\right]=\sum_{w \text { monte } V \text { sur } V^{0}} d(V, w)\left[\overline{B w^{-1} B^{-}}\right] .
$$

Le théorème suivant montre que cette expression est encore valable dans un plongement toroïdal complet et lisse de $G$.

ThÉORÈme 4.13. - Soit $X$ un plongement torö̈dal complet et lisse de $G$. Soit $V$ dans $\mathcal{V}$. Alors, dans $\mathrm{H}_{T \times\{1\}}^{*}(X)$, on $a$ :

$$
\left[\bar{V}_{G}^{X}\right]=\sum_{\substack{w \in W \\ w \text { monte } V \operatorname{sur} V^{0}}} d(V, w)\left[\overline{B w^{-1} B^{-}}\right] .
$$

Démonstration. - Comme au lemme 4.12, notons $c$ le membre de droite de l'égalité du théorème. Soit $Z_{1}, \ldots, Z_{k}$ les orbites fermées de $G \times G$ dans $X$. Considérons pour tout $i=1, \ldots, k$ le morphisme de restriction $\iota_{i}: \mathrm{H}_{T \times\{1\}}^{*}(X) \rightarrow \mathrm{H}_{T \times\{1\}}^{*}\left(Z_{i}\right)$ et le morphisme

$$
I: \mathrm{H}_{T \times\{1\}}^{*}(X) \longrightarrow \prod_{i=1}^{k} \mathrm{H}_{T \times\{1\}}^{*}\left(Z_{i}\right), \quad \delta \longmapsto\left(\iota_{i}(\delta)\right)_{i=1, \ldots, k} .
$$

D'après la proposition 4.2 , seules les orbites fermées de $G \times G$ dans $X$ contiennent des point fixes de $T \times\{1\}$. Mais le théorème de localisation (voir $[6]$ ) montre que $I$ est injective. Il suffit alors de montrer que $I\left(\left[\bar{V}_{G}^{X}\right]\right)=I(c)$. Or d'après le lemme 4.12, on a

$$
I\left(\left[\bar{V}_{G}^{X}\right]\right) \cup I\left(\left[Z_{i}\right]\right)=I(c) \cup I\left(\left[Z_{i}\right]\right), \quad \forall i=1, \ldots, k .
$$

Il suffit alors de montrer que pour tout $i=1, \ldots, k$, l'application

$$
\mathrm{H}_{T \times\{1\}}^{*}\left(Z_{i}\right) \longrightarrow \mathrm{H}_{T \times\{1\}}^{*}\left(Z_{i}\right), \quad d \longmapsto \iota_{i}\left(\left[Z_{i}\right]\right) \cup d
$$

est injective. Jusqu'à la fin de la démonstration, on fixe un indice $i$ entre 1 et $k$. Posons pour alléger les notations $Z:=Z_{i}$ et $\iota:=\iota_{i}$.

Considérons le fibré $\mathcal{N}_{Z, X}$ normal à $Z$ dans $X$. Remarquons que $\mathcal{N}_{Z, X}$ est naturellement muni d'une $G \times G$-linéarisation. De plus, $\iota(Z)$ est la classe de Chern de degré maximal et $T \times\{1\}$-équivariante de $\mathcal{N}_{Z, X}$. Si $\mathcal{M}$ est un fibré vectoriel $T$-linéarisé sur une $T$-variété, on note $c^{\max }(\mathcal{M})$ la classe de Chern de degré maximal de $\mathcal{M}$ et $c_{T}^{\max }(\mathcal{M})$ sa classe $T$-équivariante. Si $\mathcal{M}$ est un fibré en droites, nous omettrons le max en exposant.

Si $\gamma$ est un caractère de $B$, on note $\mathcal{L}_{\gamma}$ le produit fibré $G \times_{B} \mathbb{C}$, où $B$ agit sur $\mathbb{C}$ par le caractère $\gamma$. Alors, $\mathcal{L}_{\gamma}$ est un fibré en droites $G$-linéarisé sur $\mathcal{B}$. On identifie $Z$ à $\mathcal{B} \times \mathcal{B}$ comme dans la preuve du lemme 4.12. Si $\alpha$ et $\beta$ sont des caractères de $B$, on note $\mathcal{L}_{\alpha} \otimes \mathcal{L}_{\beta}$ le produit fibré $(G \times G) \times{ }_{B \times B} \mathbb{C}$, où $B \times B$ 
agit sur $\mathbb{C}$ par le caractère $(\alpha, \beta)$. Alors, $\mathcal{L}_{\alpha} \otimes \mathcal{L}_{\beta}$ est un fibré en droites $G \times G$ linéarisé sur $Z$.

Reprenons les notations de la proposition 4.1 pour $\mathcal{O}=Z$. Comme $S_{Z}$ est un plongement affine lisse et $T \times T$-équivariant de $T$, la proposition 4.1 montre qu'il existe des caractères $\alpha_{1}, \ldots, \alpha_{r}$ de $B$ tels que $\bigoplus_{j=1}^{r} \mathcal{L}_{\alpha_{j}} \otimes \mathcal{L}_{-\alpha_{j}}$ coïncide avec $\mathcal{N}_{Z, X}$ sur l'orbite ouverte de $B \times B^{-}$dans $Z$. Comme ces deux fibrés sont $G \times G$-linéarisés et $Z$ est une orbite de $G \times G$, on en déduit que $\mathcal{N}_{Z, X}$ est isomorphe à $\bigoplus_{j=1}^{r} \mathcal{L}_{\alpha_{j}} \otimes \mathcal{L}_{-\alpha_{j}}$ en tant que fibré vectoriel $G \times G$-linéarisé.

On peut maintenant calculer $\iota(Z)$ :

$$
\begin{aligned}
\iota(Z) & =c_{T \times\{1\}}^{\max }\left(\mathcal{N}_{Z, X}\right)=c_{T \times\{1\}}^{\max }\left(\bigoplus_{j=1}^{r} \mathcal{L}_{\alpha_{j}} \otimes \mathcal{L}_{-\alpha_{j}}\right) \\
& =\prod_{j=1}^{r} c_{T \times\{1\}}\left(\mathcal{L}_{\alpha_{j}} \otimes \mathcal{L}_{-\alpha_{j}}\right) \\
& =\prod_{j=1}^{r} c_{T \times\{1\}}\left(\left(\mathcal{L}_{\alpha_{j}} \otimes \mathcal{L}_{0}\right) \otimes\left(\mathcal{L}_{0} \otimes \mathcal{L}_{-\alpha_{j}}\right)\right) \\
& =\prod_{j=1}^{r}\left(c_{T}\left(\mathcal{L}_{\alpha_{j}}\right) \otimes 1+1 \otimes c\left(\mathcal{L}_{-\alpha_{j}}\right)\right) .
\end{aligned}
$$

Or, $\mathrm{H}_{T}^{*}(\mathcal{B})$ est un $\mathrm{H}_{T}^{*}(p t)$-module libre. En particulier, $c_{T}\left(\mathcal{L}_{\alpha_{j}}\right) \otimes 1$ n'est pas diviseur de zéro dans l'anneau $\mathrm{H}_{T \times\{1\}}^{*}(Z)$. Par ailleurs, $1 \otimes c\left(\mathcal{L}_{-\alpha_{j}}\right)$, qui appartient à $\mathrm{H}_{T \times\{1\}}^{*}(Z)$, est nilpotent. Mais alors, $c_{T}\left(\mathcal{L}_{\alpha_{j}}\right) \otimes 1+1 \otimes c\left(\mathcal{L}_{-\alpha_{j}}\right)$ n'est pas diviseur de zéro. On en déduit que $\iota(Z)$ n'est pas un diviseur de zéro dans $\mathrm{H}_{T \times\{1\}}^{*}(Z)$. Le théorème suit.

REMARQUE. - Il peut être étonnant de remarquer que la classe $\left[\bar{V}_{G}^{X}\right]$ est une combinaison linéaire de classes d'adhérences d'orbites de $B \times B^{-}$qui rencontrent $G$, bien que ces adhérences n'engendrent pas la cohomologie $T \times\{1\}$ équivariante de $X$.

\section{Un exemple d'adhérence d'orbite de $B$ dans un plongement de $\mathcal{H}$}

Nous donnons ici un exemple qui répond par la négative à une question posée par M. Brion (voir [4] ; après la prop. 6) à propos du lieu singulier des adhérences d'orbites d'un sous-groupe de Borel dans un plongement lisse de $\mathcal{H}$.

Soient $G=\operatorname{SL}(4)$ et $V \simeq \mathbb{C}^{4}$ le $G$-module standard. Alors $G$ agit par changement de variable sur l'espace vectoriel $\mathbb{C}[V]_{2}$ des polynômes homogènes de degré 2 sur $V$. Notons $\left(e_{1}, \ldots, e_{4}\right)$ la base canonique de $V$ et $\left(x_{1}, \ldots, x_{4}\right)$ sa base duale. Posons $q_{0}=x_{1}^{2}+\cdots+x_{4}^{2} \in \mathbb{C}[V]_{2}$. Soit $H$ le stabilisateur dans $G$ de $\left[q_{0}\right] \in \mathbb{P}\left(\mathbb{C}[V]_{2}\right)$. Alors, $\mathcal{H}=G \cdot\left[q_{0}\right]$ est l'ensemble des quadriques non dégénérées de $\mathbb{P}^{3}$.

Soit $B$ le sous-groupe de Borel de $G$ constituée des matrices triangulaires supérieures. Alors, $B$ est opposé à $H$ (en particulier, $H$ est sphérique). Les représentants contenant $B$ des classes de conjugaison de sous-groupes paraboliques

TOME $132-2004-\mathrm{N}^{\mathrm{O}} 4$ 
minimaux de $G$ sont :

$$
P_{1}=\left(\begin{array}{c|c}
* * & * \\
* * & * \\
\hline 0 & * * \\
0 & *
\end{array}\right), \quad P_{2}=\left(\begin{array}{c|c|c}
* & * & * \\
\hline 0 & * * & * \\
\hline 0 & * * & * \\
\hline 0 & *
\end{array}\right), \quad P_{3}=\left(\begin{array}{cc|c}
* * & * \\
0 * & \\
\hline 0 & * * \\
\hline & * *
\end{array}\right) .
$$

D'après [11], le graphe $\Gamma(G / H)$ est représenté par la figure 2 .

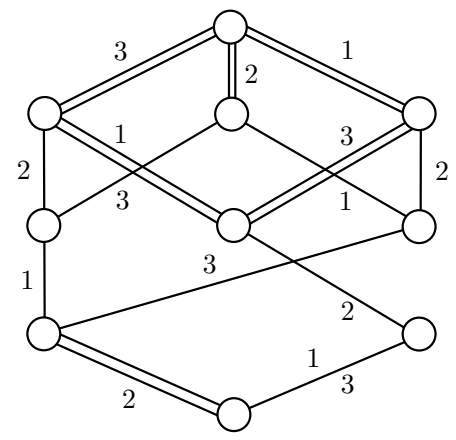

Figure 2. Le graphe $\Gamma(G / H)$

L'orbite fermée $V_{\mathcal{H}}$ de $B$ dans $\mathcal{H}$ est :

$$
\left\{[q] \in \mathcal{H} \subset \mathbb{P}\left(\mathbb{C}[V]_{2}\right): q\left(e_{1}, e_{i}\right)=0, i=1,2,3, q\left(e_{2}, e_{2}\right)=0\right\} .
$$

En fait, $\mathcal{H}$ est un PSL(4)-espace homogène symétrique. Considérons le plongement magnifique $Y$ de $\mathcal{H}$ construit par De Concini et Procesi dans [5]. On peut montrer que $Y$ est l'unique plongement toroïdal complet de $\mathcal{H}$ et contenant une unique orbite fermée $Z$. De plus, De Concini et Procesi ont montré que $Y$ était lisse. Considérons l'adhérence $\bar{V}_{\mathcal{H}}^{Y}$ de $V_{\mathcal{H}}$ dans $Y$. On peut maintenant énoncer le

THÉORÈme 5.1. - Avec les notations ci-dessus, $\bar{V}_{\mathcal{H}}^{Y} \cap Z$ a une composante irréductible constituée de points singuliers de $\bar{V}_{\mathcal{H}}^{Y}$.

Démonstration. - Soit $T$ le tore maximal de $G$ constitué des matrices diagonales. Soit $\left(\alpha_{1}, \alpha_{2}, \alpha_{3}\right)$ les racines simples de $(B, T)$. Posons :

$$
Y_{Z, B}:=\{y \in Y: \overline{B \cdot y} \supset Z\} .
$$

Notons $S$ l'adhérence de $T \cdot\left[q_{0}\right]$ dans $Y_{Z, B}$. Il est montré dans [5] que la $T$ variété $S$ est isomorphe à $\mathbb{A}^{3}$ sur lequel $T$ agit linéairement par les poids $2 \alpha_{1}, 2 \alpha_{2}$ et $2 \alpha_{3}$. Fixons un tel isomorphisme $\xi: \mathbb{A}^{3} \rightarrow S$. Soit $U$ le radical unipotent de $B$. Alors (voir [5]) l'application

$$
\Theta: U \times \mathbb{A}^{3} \longrightarrow Y_{Z, B}, \quad(u, \tau) \longmapsto u \cdot \xi(\tau)
$$

est un isomorphisme. De plus, $\Theta^{-1}\left(Y_{Z, B} \cap Z\right)=U \times\{0\}$. 
Soit $s_{1}, s_{2}$ et $s_{3}$ les réflexions simples du groupe de Weyl $W$ de $T$ associées respectivement à $P_{1}, P_{2}$ et $P_{3}$. Considérons l'élément $w=s_{2} s_{1} s_{3} s_{2}$ de $W$ et fixons le représentant $\tilde{w}$ de $w$ suivant :

$$
\tilde{w}=\left(\begin{array}{c|cc}
0 & 1 & 0 \\
& 0 & 1 \\
\hline 1 & 0 & 0 \\
0 & 1 & 0
\end{array}\right) .
$$

Posons $\Omega:=w \bar{V}_{\mathcal{H}}^{Y} \cap Y_{Z, B}$. Alors, $\Omega$ est un ouvert de $w \bar{V}_{\mathcal{H}}^{Y}$ stable par $B_{w}$. Comme $\Theta$ est un isomorphisme, on a

$$
\Omega=B_{w} \cdot\left(\left(\begin{array}{ll|l}
1 & 0 & * \\
0 & 1 & * \\
\hline 0 & 1 & 0 \\
0 & 0 & 1
\end{array}\right) \cdot\left[q_{0}\right] \cap \tilde{w} V_{\mathcal{H}}\right) .
$$

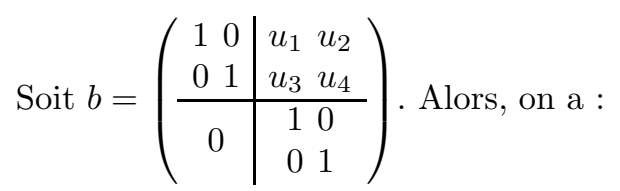

$$
\begin{aligned}
b \cdot\left[q_{0}\right] \in \tilde{w} V_{\mathcal{H}} & \Longleftrightarrow \tilde{w}^{-1} b\left[q_{0}\right] \in V_{\mathcal{H}} \\
& \Longleftrightarrow\left[\begin{array}{l}
\left(1+u_{3}^{2}+u_{1}^{2}\right) x_{1}^{2}+2\left(u_{3} u_{4}+u_{1} u_{2}\right) x_{1} x_{2}-2 u_{1} x_{1} x_{3} \\
\quad-2 u_{3} x_{1} x_{4}+\left(1+u_{2}^{2}+u_{4}^{2}\right) x_{2}^{2}-2 u_{2} x_{2} x_{3}
\end{array}\right. \\
& \left.\quad-2 u_{4} x_{2} x_{4}+x_{3}^{2}+x_{4}^{2}\right] \in V_{\mathcal{H}} \\
& \Longleftrightarrow\left\{\begin{array} { l } 
{ 1 + u _ { 3 } ^ { 2 } + u _ { 1 } ^ { 2 } = 0 , } \\
{ u _ { 3 } u _ { 4 } + u _ { 1 } u _ { 2 } = 0 , } \\
{ u _ { 1 } = 0 , 1 + u _ { 2 } ^ { 2 } + u _ { 4 } ^ { 2 } = 0 , }
\end{array} \Longleftrightarrow \left\{\begin{array}{l}
u_{1}=u_{4}=0, \\
u_{2}= \pm i, \\
u_{3}= \pm i .
\end{array}\right.\right.
\end{aligned}
$$

Si $y=\left(\begin{array}{cc|cc}1 & 0 & 0 & i \\ 0 & 1 & i & 0 \\ \hline 0 & 1 & 0 \\ 0 & 1\end{array}\right) \cdot\left[q_{0}\right]$, on en déduit que $w V_{\mathcal{H}} \cap Y_{Z, B}=U_{w} \cdot T \cdot y$.

Comme $\Theta$ est un isomorphisme, on a

$$
\Omega=w \bar{V}_{\mathcal{H}}^{Y} \cap Y_{Z, B}=U_{w} \cdot \overline{T \cdot y}^{Y_{Z, B}} .
$$

Muni de l'action de $T, Y_{Z, B}$ est isomorphe à un $T$-module. De plus, les poids de $y \in Y_{Z, B}$ pour l'action de $T$ sont

$$
2 \alpha_{1}, 2 \alpha_{2}, 2 \alpha_{3}, \alpha_{2}, \alpha_{1}+\alpha_{2}+\alpha_{3} .
$$

Comme cette famille de $\mathcal{X}^{*}(T)$ ne contient pas de sous-famille libre qui engendre le même sous-groupe de $\mathcal{X}^{*}(T)$, l'adhérence $\overline{T \cdot y} Y_{Z, B}$ n'est pas lisse. En particulier, $\xi(0)$ est un point singulier de $\overline{T \cdot y} Y_{Z, B}$. Par ailleurs, comme $\Theta^{-1}\left(Y_{Z, B} \cap Z\right)=U \times\{0\}$, on a $w \bar{V}_{\mathcal{H}}^{Y} \cap Y_{Z, B} \cap Z=U_{w} \cdot \xi(0)$. Le théorème suit aisément.

TOME $132-2004-\mathrm{N}^{\mathrm{O}} 4$ 


\section{Appendice : espaces fibrés algébriques}

N'ayant pas trouvé de référence satisfaisante dans la littérature, je démontre dans cette appendice un lemme sur les espaces fibrés algébriques au sens de J.-P. Serre [16] (voir aussi $[12, \S 4.8]$ ).

Soit $H$ un groupe algébrique affine. Soient $P$ une $H$-variété et $\pi: P \rightarrow X$ un morphisme $H$-invariant de $P$ dans une variété $X$. Le triplet $(H, P, X)$ est appelé un système fibré. On appelle espace fibré principal de base $X$ et de groupe $H$ un système fibré $(H, P, X)$ qui est localement trivial dans la topologie étale. Dans [16], J.-P. Serre dit que $(H, P, X)$ est localement isotrivial.

Soient $G$ un groupe algébrique affine et $H$ un sous-groupe fermé de $G$. Soit $F$ une $H$-variété. Considérons sur $G \times F$ l'action de $H$ donnée par la formule $h \cdot(g, f)=\left(g h^{-1}, h f\right)$. S'il existe une variété $X$ et un morphisme $\pi: G \times F \rightarrow X$ tels que $(H, G \times F, X)$ soit un espace fibré principal, cette variété et ce morphisme sont uniques (voir [16, preuve de la prop.4]) On appelle alors $X$ le produit fibré de $G$ par $F$ au-dessus de $H$ et on le note $G \times{ }_{H} F$.

Dans le cas où le système fibré $(H, G, G / H)$ est localement trivial dans la topologie de Zariski, il est facile de voir que $G \times_{H} F$ existe. Ceci s'applique par exemple lorsque $H$ est résoluble et connexe (voir [15]).

Dans une autre direction, les propositions 3 et 4 de [16] (ou le théorème 4.19 de [12]) montrent que $G \times{ }_{H} F$ existe si $F$ est quasiprojective.

Si le produit fibré $G \times{ }_{H} F$ existe, l'action de $G$ sur $G \times F$ par multiplication à gauche sur le premier facteur induit une action algébrique de $G$ sur $G \times_{H} F$. De plus, la projection de $G \times F$ sur $G$ induit un morphisme $G$-équivariant $G \times{ }_{H} F \rightarrow G / H$. Le lemme suivant montre que $G \times{ }_{H} F$ est l'unique $G$-variété qui soit munie d'un morphisme $G$-équivariant sur $G / H$ dont la fibre au-dessus de $H / H$ soit $F$.

Lemme 6.1. - Soit $X$ une G-variété et $\phi: X \rightarrow G / H$ un morphisme $G$ équivariant. Posons $F=\phi^{-1}(H / H)$ et considérons $\pi: G \times F \rightarrow X$ définie par $(g, x) \mapsto g \cdot x$. Alors, le triplet $(H, G \times F, X)$ est un espace fibré principal de base $X$ et de groupe $H$. Autrement dit, $G \times_{H} F$ existe et est isomorphe à $X$.

Démonstration. - Il est évident que $(H, G \times F, X)$ est un système fibré. Il suffit donc de montrer qu'il est localement trivial dans la topologie étale. D'après [16, prop. 2], il suffit de montrer les propriétés (FP) et (SL) ci-après :

(FP) Si $(g, x)$ et $\left(g^{\prime}, x^{\prime}\right)$ sont deux éléments de $G \times F$ tels que $g \cdot x=g^{\prime} \cdot x^{\prime}$ alors il existe un unique $h \in H$ tel que $\left(g^{\prime}, x^{\prime}\right)=h \cdot(g, x)$. De plus, l'application qui, $\grave{a}$ un tel couple $\left((g, x),\left(g^{\prime}, x^{\prime}\right)\right)$ fait correspondre l'élément $h$ est un morphisme de la sous-variété $T$ de $(G \times F) \times(G \times F)$ où elle est définie dans le groupe $H$.

(SL) Pour tout $x \in X$, il existe un revêtement non ramifié $f: U^{\prime} \rightarrow U$ d'un voisinage ouvert $U$ de $x$, et un morphisme $s: U^{\prime} \rightarrow P$ tels que $\pi \circ s=f$ sur $U^{\prime}$.

La condition (FP) se vérifie immédiatement (on a $h=g^{\prime-1} g$ ).

BULletin DE LA SOCiÉtÉ MATHÉMATIQUE DE FRANCE 
Soit $x \in X$. Notons $p: G \rightarrow G / H$ la projection canonique. D'après [16, prop. 3], le triplet $(H, G, G / H)$ est un espace fibré principal. En particulier, il existe un revêtement non ramifié $\psi: \Omega^{\prime} \rightarrow \Omega$ d'un voisinage ouvert $\Omega$ de $\phi(x)$ et $\sigma: \Omega^{\prime} \rightarrow G$ tels que $\psi=p \circ \sigma$. Posons

$$
U^{\prime}=\left\{(\omega, x) \in \Omega^{\prime} \times X: \psi(\omega)=\phi(x)\right\} .
$$

Alors, par $[16,1.3 . \mathrm{d}]$, la projection de $\Omega^{\prime} \times X$ sur $X$ induit un revêtement non ramifié $f: U^{\prime} \rightarrow \phi^{-1}(\Omega)$. Posons

On vérifie que $\pi \circ s=f$.

$$
s: U^{\prime} \longrightarrow G \times F, \quad(\omega, x) \longmapsto\left(\sigma(\omega), \sigma(\omega)^{-1} \cdot x\right) .
$$

\section{BIBLIOGRAPHIE}

[1] Brion (M.) - Quelques propriétés des espaces homogènes sphériques, Manuscripta Math., t. 55 (1986), no. 2, pp. 191-198.

[2] _ Variétés sphériques, Notes de la session de la SMF Opérations hamiltoniennes et opérations de groupes algébriques, Grenoble; http/ /www-fourier.ujf-grenoble.fr/〜mbrion/spheriques.ps, 1997.

[3] , The behaviour at infinity of the Bruhat decomposition, Comment. Math. Helv., t. 73 (1998), no. 1, pp. 137-174.

[4] - On orbit closures of spherical subgroups in flag varieties, Comment. Math. Helv., t. 76 (2001), no. 2, pp. 263-299.

[5] De Concini (C.) \& Procesi (C.) - Complete symmetric varieties, in Invariant theory (Montecatini, 1982), Springer, Berlin, 1983, pp. 1-44.

[6] Hsiang (W.) - Cohomology theory of topological transformation groups, Springer-Verlag, New York, 1975.

[7] Humphreys (J.E.) - Linear algebraic groups, Springer Verlag, New York, 1975.

[8] Knop (F.) - The Luna-Vust theory of spherical embeddings, in Proceedings of the Hyderabad Conference on Algebraic Groups (Hyderabad, 1989), Manoj Prakashan, Madras, 1991, pp. 225-249.

[9] t. 70 (1995), no. 2, pp. 285-309.

[10] Mumford (D.), Fogarty (J.) \& Kirwan (F.) - Geometric invariant theory, $3^{\text {e }}$ éd., Springer Verlag, New York, 1994.

[11] PIN (S.) - Sur les singularités des orbites d'un sous-groupe de Borel dans les espaces symétriques, Thèse, Université Grenoble I, 2001, http: //www-fourier.ujf-grenoble.fr/THESE/these】_daterev.html.

[12] Popov (V. L.) \& Vinberg (亡̀.B.) - Invariant Theory, in Algebraic Geometry IV (Parshin (A.N.) \& Shafarevich (I.R.), éds.), Encyclopedia of Mathematical Sciences, vol. 55, Springer-Verlag, 1991, pp. 123-284.

TOME $132-2004-\mathrm{N}^{\mathrm{O}} 4$ 
[13] Ressayre (N.) - Quotients of group completions by spherical subgroups, J. Algebra, t. 265 (2003), no. 1, pp. 1-44.

[14] Richardson (R.W.) \& Springer (T.A.) - The Bruhat order on symmetric varieties, Geom. Dedicata, t. 35 (1990), no. 1-3, pp. 389-436.

[15] Rosenlicht (MAxwell) - Some basic theorems on algebraic groups, Amer. J. Math., t. 78 (1956), pp. 401-443.

[16] Serre (J.-P.) - Espaces fibrés algébriques, in Séminaire C. Chevalley; $2^{e}$ année : 1958. Anneaux de Chow et applications, Secrétariat mathématique, E. N. S. Paris, 1958, Exp. no 1, pp. 1-37.

[17] Springer (T.A.) - Linear algebraic groups, $2^{\mathrm{e}}$ éd., Birkhäuser Boston Inc., Boston, MA, 1998.

[18] Intersection cohomology of $B \times B$-orbit closures in group compactifications, J. Algebra, t. 258 (2002), no. 1, pp. 71-111.

[19] VinBerg (亡̀.B.) - Complexity of actions of reductive groups, Funktsional. Anal. i Prilozhen., t. 20 (1986), no. 1, pp. 1-13, 96.

[20] Wolf (J.A.) - Admissible representations and geometry of flag manifolds, in The Penrose transform and analytic cohomology in representation theory (South Hadley, MA, 1992), American Mathematical Society, Providence, RI, 1993, pp. 21-45. 